\title{
FIVE NEW CLAVATE-STIGMA WALTHERIA SPECIES ENDEMIC TO BRAZILIAN CERRADO (MALVACEAE S. L., BYTTNERIOIDEAE, HERMANNIEAE)
}

\author{
Janice G. Saunders
}

Instituto de Botánica Darwinion (ANCEFN-CONICET), Labarden 200, Casilla de Correo 22, B1642HYD San Isidro, Buenos Aires, Argentina; jsaunders@darwin.edu.ar (author for correspondence).

\begin{abstract}
Saunders, J. G. 2021. Five new clavate-stigma Waltheria species endemic to Brazilian Cerrado (Malvaceae s. 1., Byttnerioideae, Hermannieae). Darwiniana, nueva serie 9(1): 5-30.

Five new species are described and illustrated: Waltheria hatschbachii, W. hoehnei, both endangered for extinction, $W$. coriacea, $W$. flavovirens and $W$. matogrossensis. All are endemic to Brazil, in Cerrado s. 1. associated with the Brazilian Plateau in N, NE, CW, and SE Brazil. Their affinities and the distinctness from $W$. ferruginea $\mathrm{s}$. 1. are discussed, and they are associated and compared with $W$. ferruginea s. str., from cerrado, $W$. brachypetala, from caatinga, $W$. biribiriensis and $W$. terminans, both from campo rupestre. The geographic distribution of all nine species is illustrated, and a new identification key for these and for the two sections of Waltheria is provided. All nine species share a clavate stigma in pin (or thrum) flowers, that morphotype given earlier as distinguishing one of three main species groups of the genus, and a new character, the ferruginea type stipitate-stellate trichome, illustrated here. Subconduplicate petals are first reported in the tribe Hermannieae, often keyed by flat petals. Waltheria is first signaled here to present the same enigmatic biogeographical pattern noted before for Triraphis (Poaceae) between Chapada dos Veadeiros, Africa and Australia. Other new taxonomic characters for Waltheria are given.
\end{abstract}

Keywords. Biogeography; Brazil; Byttnerioideae; cerrado; conservation; Hermannieae; taxonomy; Waltheria.

Resumen. Saunders, J. G. 2021. Cinco especies nuevas con estigma clavado de Waltheria, endémicas del Cerrado de Brazil (Malvaceae s. 1., Byttnerioideae, Hermannieae). Darwiniana, nueva serie 9(1): 5-30.

Se describen e ilustran cinco nuevas especies: Waltheria hatschbachii, W. hoehnei, ambas en peligro de extinción, $W$. coriacea, $W$. flavovirens y $W$. matogrossensis, todas endémicas del Cerrado s. 1 . de Brasil, asociadas al Planalto, en el N, NE, CO y SE. Se describen, ilustran y discuten sus afinidades, su distinción con $W$. ferruginea s. 1. y se asocian y se comparan con $W$. ferruginea s. str., del cerrado; $W$. brachypetala, de la caatinga; $W$. biribiriensis y $W$. terminans, ambas del campo rupestre. Se presentan las distribuciones geográficas de las nueve especies, una clave para diferenciarlas y una clave con las dos secciones de Waltheria. Todas estas especies comparten las flores longistilas (o brevistilas) con un estigma clavado, morfotipo señalado previamente para diferenciar uno de los tres principales grupos del género, y la presencia de tricomas estrellados, de base estipitada, del tipo ferruginea, un nuevo carácter aquí ilustrado. Los pétalos subconduplicados, se registran por primera vez en la tribu Hermannieae, a menudo señalados como pétalos planos. Se informa sobre la particular y enigmática distibucion geográfica de Waltheria en Chapada dos Veadeiros, África y Australia, compartida con Triraphis (Poaceae). Otros caracteres taxonómicos nuevos para Waltheria son presentados.

Palabras claves. Biogeografía; Brasil; Byttnerioideae; cerrado; conservación; Hermannieae; taxonomía; Waltheria. 


\section{INTRODUCTION}

Waltheria L. is the only one-carpellate genus of the tribe Hermannieae, a natural coherent tribe. The tribe was placed in the Sterculiaceae, but is now generally placed within the Byttnerioideae sensu Whitlock et al. (2001), subfamily of an expanded Malvaceae.

This work involves species whose individuals were previously determined as Waltheria ferruginea A. St.-Hil. since the last generic Brazilian revision done by Schumann (1886). Schumann, treating 20 Brazilian Waltheria species, states that he did not see original material of Saint-Hilaire's Waltheria ferruginea, but that according to its description and illustration, considered it to be the same species as $W$. brachypetala Turcz., the only species examined then (Saunders, 1995). These two species he combined using the earlier name $W$. ferruginea and that name was later also misapplied to other new species. A phylogenetic study of 60 species (Saunders, unpubl. data with results discussed in Saunders, 1995) and a monograph dissertation with taxonomic treatment of 57 species of Waltheria (Saunders, 1995) identified nine species within the former broader concept of $W$. ferruginea sensu Schumann: $W$. ferruginea, $W$. brachypetala, and seven other new species, described and illustrated in that dissertation but not effectively published. Two of these new species were recently published by Coutinho et al. (2020): W. biribiriensis J.G. Saunders ex T.S. Coutinho \& Colli-Silva and $W$. terminans J.G. Saunders ex T.S. Coutinho \& ColliSilva. In this contribution, the remaining five of those seven species new to science are effectively published. A larger phylogenetic study of all nine species of the $W$. ferruginea $\mathrm{s}$. str. allies, the focus of intensive investigation for the last several years, will be presented in another work.

Here, Waltheria ferruginea $\mathrm{s}$. str. is only applied to the original species endemic to Minas Gerais from the vicinity of Grão Mogol and Cristalia. That species was illustrated and redescribed by Cristóbal \& Saunders (2006) based on material collected by this author near Grão Mogol. It was separated as distinct from $W$. brachypetala by this author in the Flora of Mandiba (Amorim et al., 2009), based upon the long velutinous overlayer present on calyx and bract of that species. W. brachypetala was redescribed there without using characters from the original description of $W$. ferruginea, in contrast to using them as was done by Schumann (1886).

This work presents the diagnoses, descriptions, and illustrations for the 5 new Waltheria species endemic to Brazilian cerrado s. 1. Their affinities and the reason for distinct species status are discussed. They are associated and compared with $W$. ferruginea, from cerrado, $W$. brachypetala, from caatinga, $W$. biribiriensis and $W$. terminans, both from campo rupestre. The geographic distribution of all nine species is illustrated, and new identification keys for these and for the two previous formally described sections of Waltheria are provided. All nine species are placed in one of three main informal groups of the genus described earlier based upon pin stigma type (Saunders, 1993), the clavate pin stigma group. Within that group they are united to each other by the presence of the ferruginea type stipitate-stellate trichome, a new character first described here and illustrated as such. Subconduplicate petals are first reported here in a tribe (Hermannieae) usually keyed by presence of flat petals to oppose those with cucullate-ligulate petals found in Theobromeae or Byttnerieae. Other new taxonomic characters for Waltheria are also presented. The misapplication of the name $W$. polyantha K. Schum. is discussed. Waltheria is here first signaled to present an enigmatic biogeographical pattern noted before for Triraphis R. Br. (Poaceae) between Chapada dos Veiadeiros, Africa and Australia.

\section{MATERIAL AND METHODS}

Traditional taxonomic methods and concepts were used to apply to the problem of plants labeled as Waltheria ferruginea or resembling it, studied within a broader dissertation study that focused on Brazilian species for a monograph of the entire genus. Fieldwork guided by Brazilian taxonomists in seven states of Brazil during February to May of 1990 provided photographs, data, observations and successive populational samples for 23 of the 30 Waltheria species for Brazil. This included seven species of the $W$. ferruginea allies, and type localities for $W$. ferruginea and $W$. brachypetala. Later, assembled relevant herbarium material for this study was sorted by visual inspection into different groups based upon gross morphology. Representative samples of each group were compared within and between groups 
in a morphological comparative study, including 34 basic measurements per specimen, most using a binocular microscope with a micrometer inserted, except for leaves and inflorescence size done with a ruler. It included distribution of trichome types, and comparison of shapes of stipules, petioles, leaves, bracteoles, bracts, calyx, calyx lobes, calyx veins, petals, androecium, pistils, stigma, capsule, and seed using sketches, camera lucida drawings, or representive dissected material, dry for hairs, or rehydrated flower parts using Pohl's solution (Pohl, 1965), later mounted in Hoyer's solution (Radford et al., 1974). Measurement ranges for structures between floral morphs and between species were compared in tables. One representative of each species was scored for over 160 morphological characters, including trichome type and distribution on plant parts. If a character state was known to vary within the species it was scored as a polymorphism. The preliminary phylogenetic analysis of the entire genus (unpubl. data, Saunders results summarized and discussed in Saunders, 1995) provided important character hierarchy to rank and evaluate species differences for this group. This basic morphological study was used to conclude if differences between groups in $W$. ferruginea allies were either by mostly non-reproductive structures and clinal or varietal with introgression, or if numerous constant differences for taxonomically important floral and fruit features were present, if autapomorphies were present, and if groups with significantly different morphology demonstrated allopatric species distribution for identifiable discrete species status. The original descriptions, original collections, important taxonomic revisions were examined before, during and after the study to write descriptions and ultimately assign correct names, select and designate types and lectotypes for $W$. brachypetala and $W$. ferruginea and the new species. Keys reflect easy characters, not all the important phylogentic ones. Material examined is from dried bulk samples, FAA preserved samples and herbarium material from the following herbaria: A, ARIZ, B, BBS, BH, BHCB, BKL, BM, BR, C, CAS-DS, CAY, CEPEC, CTES, DAV, DNA, E, F, FCQ, G, GH, GUA, HAL, HB, HUEFS, INPA, JE, K, KW, LE, LIL, LL, LPB, M, MA, MBM, MEXU, MICH, MO, MSC, NY, P, PR, R, RB, S, SI, SING, SP, SPF, TEX, TI, U, UB, UCR, UFMT, UPS, US, WIS and W (Thiers, 2020).

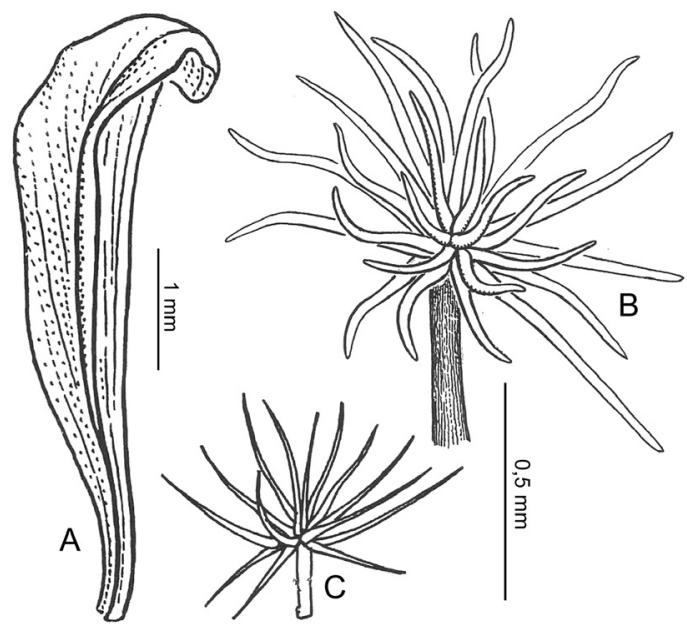

Fig. 1. Subconduplicate petal and Ferruginea type stipitatestellate trichome (FSST). A, subconduplicate petal of $W$. ferruginea. B, FSST from stem of $W$. ferruginea. $\mathbf{C}$, FSST from adaxial leaf of $W$. biribiriensis. A, B, from Saunders et al. 3079. C, from Saunders et al. 3167. Illustration: F. Rojas.

Terminology used: "crested inflorescences" describe a secund, crestlike, erect inflorescence along the branch over distichous, horizontal to reclining leaves, best noted by field observations. Calyx shape is as Schumann (1886). "Subduplicate" petals are half-closed longitudinally, at times thickened dorsally at base, up to a reflexed to recurved apex more or less flat (Fig. 1A). Seed chalaza is substituted for "strophiole", used earlier by the author. Terminology for indument is given for 3-tiered layers on vegetative parts in descending order, and progressively shorter height and/or size for stellate trichomes in indument: hirsute (firm), hirsutulous (firm), woolly-tomentose, velutinous, hirsutulous (firm), tomentose, tomentulose, villulose, tomentellous, pannose. Traditional definitions for vestiture are used (Lawrence, 1951: 746, Figs. 308-309), adding hirsutulous: short hirsute; tomentellous: diminuitive tomentose; tomentulose: short tomentose; villulose: short villous.

Cerrado physiognomy types are adapted from Oliveira-Filho \& Ratter (2002: 96): Cerrado s. str., "low open woodland with $\geq 30 \%$ crown cover of trees 3-8 m tall, but a fair amount of herbaceous groundlayer"; campo cerrado, open grassland, less than $30 \%$ low scattered shrub cover; capão de cerrado, "isolated, hilltop forest (Hensold, 1988: 43); 
cerradão, " $50 \%-90 \%$ closed crown cover trees often 8-12 $\mathrm{m}$ tall to tall subforest shading out herbaceous layer" in moister soils; carrascal, secondary cerrado; campo rupestre, rocky grasslands at high altitudes; campo limpo, no shrubs; caatinga, semiarid open dry deciduous thorn scrub shrub or arboreal forest at low altitudes.

Darwin's terms of "pin" and "thrum" are used for longistylous and brevistylous flowers respectively, for these distylous (heterostylous) Waltheria species. Pin flowers have one long style and 5 shorter stamens. Thrum flowers have 5 long stamens and one short style. The pistil is usually in reciprocal length to stamen lengths between pin and thrum flowers, each floral morphological type exclusive to one plant. In distylous species, isoplethy, that is an equal proportion of pin-flowered plants and thrumflowered plants in a native locality population (commonly of 10-30 individuals), indicates that the heteromorphic sporophytic incompatibility system, found only in distylous species studied, is presumably present.

Diurnal flowers open for more than 7 hours. Diurnal flowers were arbitrarily defined for the genus as those with petals closing after 7 hours in midafternoon to early evening, from a morning opening (8-10 am typically), in contrast to matutinal flowers arbitrarily defined as closing for the day after 3 to 7 hours, usually closing, depending on the species in the genus, from mid-morning until early afternoon. Flower petals do not open after one day, petals remain wilted, but some are still visited by some insects.

Each new species was assessed for conservation status using the IUCN criteria (2019).

\section{RESULTS}

\section{Taxonomic treatment}

Waltheria coriacea J.G. Saunders, sp. nov. TYPE. Brazil, Bahia, $60 \mathrm{~km}$ E of Mimoso on BR-242 Mimoso-Barreiras, (33 km W of Barreiras), ca. $12^{\circ} 07$ 'S, $45^{\circ} 25$ 'W, 18-II-1990, J. G. Saunders, L. Breyer \& G. Eiten 2071 (holotype UB 217282! [thrum]; isotypes CEPEC 71876! [thrum], CTES 254948! [thrum], barcode K 000381059 ! [thrum], TEX 00557325!, [thrum], TEX 00557326! [thrum]). Figs. 2, 3A.
Diagnosis. Differs from $W$. brachypetala by coriaceous, lustrous leaves; inflorescence soon very lax; bracts subequal, 3-4.5 mm long, narrowly triangular; thrum stamens unequal, tube uneven; short stipitate stellate trichomes at stigma base and on style apex of both pin and thrum pistils.

Shrubs 1-3 m tall. Ferruginea type stipitate-stellate trichomes present on vegetative parts, abaxial calyx: stipes ochre to red, $0.2 \mathrm{~mm}$; stellate rays ca. 20 , ca. $0.1 \mathrm{~mm}$, ferrugineous. Branches distally ferrugineoustomentose, pannose, stellate, stipitate-stellate; older stem surface castaneous, brown, ridges anastomose, transversely oblong lenticels present; internodes 10-70 mm. Stipules narrowly triangular, 1-4.7 mm, base truncate, both sides densely hirsute, stellate, some stipitate, adaxial also with simple trichomes, glands obovoid. Leaves distichously arranged, evenly distributed, horizontal to reclining; petioles 5-13 $\times$ 1-16 mm, ridged; laminas discolorous, coriaceous, variable, usually ovate-oblong or elliptic, ovate, narrowly elliptic, circular, obovate, $2.3-7.5 \times 2-3.6 \mathrm{~cm}$, usually apex obtuse, acute to rounded, base usually rounded, cuneate to subcordate for 1-2 $\mathrm{mm}$, insertion planar, margins serrate, teeth $1.2-3 \times 0.6-1.2 \mathrm{~mm}$, adaxial ferrugineous, hispidulous, ephemeral pannose, stellate, stipitate-stellate, surface asperous, lustrous, subrugose, rufous dry, bright green fresh; abaxial olive, tomentose, pannose, ferrugineous stipitatestellate on veins, 4-8 lateral vein pairs above basal 3-7 palmate veins. Inflorescence axillary, crested, cymose, soon very lax, 2.5-8 cm, 16-65-flowered, floral pairs widely separated. Bract stipe $0.5-1 \mathrm{~mm}$; bracts 4 per floral pair, persistent, subequal, connate at $0-1 \mathrm{~mm}$, 3-4.5 $\times 0.6-1.7 \mathrm{~mm}$, narrowly triangular, both sides covered with stellate-hirsute trichomes, adaxial also with pyriform glandular. Bracteoles 2-3, connate, 1.8$4.5 \mathrm{~mm}$. Pedicels $0-0.1 \mathrm{~mm}$. Flowers distylous, ca. $3 \mathrm{~mm}$ diam. corolla. Calyx turbinate-clavate, 5-ribbed, 5-dentate, 4.5-6.7 × 2.8-3.7 mm; base articulate; teeth deltate, $0.9-1.7 \mathrm{~mm}$, interior villulose, trichomes simple; tube interior hirsute, base nectaries to $0.6 \mathrm{~mm}$; exterior calyx tomentellous, stellate, stipitatestellate. Petals (4-)5, lemon yellow, spathulate, subconduplicate, 3-5.2 × 1-1.9 mm, emarginate, rounded, eciliate, subglabrous, adaxial surface with few trichomes, rays 2 , base free from stamens. Thrum stamens (4-)5, unequal, glabrous, papillose, 4-5.8 mm, stamen tube uneven, 1-2.4 mm; anthers 0.9-1.2 mm. 


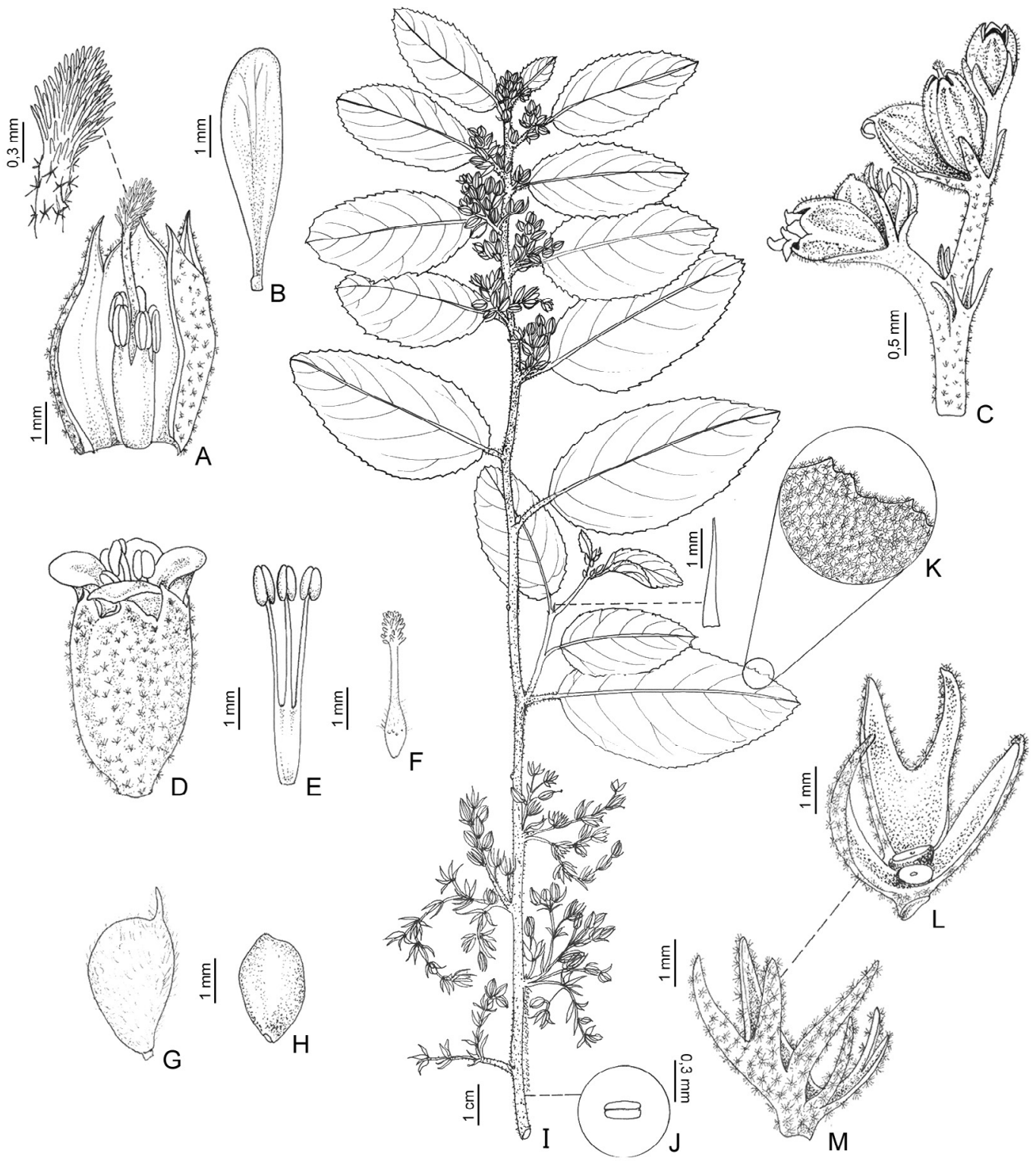

Fig. 2. Waltheria coriacea. A, pin flower, split. B, petal, adaxial flattened. C, two mature floral pairs, cymose. D, thrum flower. E, thrum stamens, 3 of 5. F, thrum pistil. G, capsule. H, seed. I, old, new flowering branch. J, transversely oblong lenticel. $\mathbf{K}$, adaxial leaf lamina. $\mathbf{L}$, two flower pedicels, four adaxial bracts. $\mathbf{M}$, inferior three bracteoles opposing superior four bracts. All from Saunders et al. 2069 pin, Saunders et al. 2062 thrum (TEX). Illustration: Y. Wilson-Ramsey.

Thrum pistil 3.2-4.4 mm; ovary 1-1.4 $\mathrm{mm}$, apex hirsute, stellate, sparse, fewer trichomes, fewer rays to base; style 1.2-2.5 $\mathrm{mm}$, hirsutulous, tomentose to stigma, stellate, a few medium long-stipitate trichomes at apex; stigma reflexed, clavate-fimbriate, oblongoid, pale green, 0.6-1.2 × 0.2-0.7 mm. Androgynophore $0.2 \mathrm{~mm}$. Pin stamens 2.9-4 mm, filaments 5, stamen tube 1.7-2.9 mm. Pin pistil4.6-7.2 mm; ovary 1-1.1 mm, densely hirsute to a base glabrous for $0.1 \mathrm{~mm}$; style distal half arcuate, recurved with stigma, moderately densely stellate, some stipitate-stellate trichomes at apex and stigma base (Fig. 4A), stipes short, 0.03$0.1 \mathrm{~mm}$; stigma clavate-fimbriate, exserted presentation excentric, bovoid, pale green, $0.5-0.8(-1.1) \times 0.2-0.7 \mathrm{~mm}$. 




Fig. 3. Field pictures of: A, Waltheria coriacea with Pepsis sp. B, W. flavovirens. C, W. matogrossensis. A, from Saunders 2085 thrum flowered plant. B, from one of 3 Saunders et al. 2053-2055 thrum flowered plants. C, from Saunders et al. 3058 thrum flowered plant. Color version at http://www.ojs. darwin.edu.ar/index.php/darwiniana/article/view/928/1204

Capsule hidden in calyx, obovoid, 5.7-6 $\times 2.5-3 \mathrm{~mm}$, apex rounded, sparser stellate, fewer rays to 2 toward base; loculicidal dehiscence incomplete. Seed brown, obovoid, 1.8-2.4 × 1.2-1.7 mm, apex rounded, striate, minutely verrucose, papillose.

Common name. "Malva de embira", Viollati et al. 251 (CTES).
Etymology. The specific epithet "coriacea" is derived from its coriaceous, leathery leaves.

Distribution and habitat. Waltheria coriacea is endemic to NE (Bahia, Maranhão), N (Tocantins), and CW (Goiás) Brazil, on the slopes of the Serra Geral de Goiás and Espigão Mestre, and disjunct to Maranhão on Serra Baleia and Tasso Fragosso. Elevations range from 320-920 m a.s.l. (Fig. 5). Waltheria coriacea is native in cerradão, cerrado woodland with open or closed forest, closed scrub cerrado woodland without open campo, in remnant cerrado from open forest with closed scrub woodland, occurring on rocky sandstone slopes, sandy white, reddish, light brown sandy soils, rarely on clay with sandy soil (G. Eiten, pers. comm.).

Observations. Waltheria coriacea and $W$. matogrossensis form a sister pair in preliminary phylogenetic analysis (unpubl. data, Saunders 1995). The stigma shapes are very similar, and both share the derived character of pin styles that are moderately to densely stellate from base to apex. A new character, presence of stipitatestellate trichomes, with a medium long stipe in $W$. matogrossensis, but short in $W$. coriacea, found in or below the stigma base in both pin and thrum flowers is a shared synapomorphy for $W$. coriacea and $W$. matogrossensis (Fig. 4A, B, respectively). Waltheria coriacea can be distinguished from all the species in the $W$. ferruginea allies by its coriaceous, lustrous leaves, and by its inflorescence soon very lax rather than rarely lax in age, as in one example of $W$. matogrossensis (see comments there), and from $W$. matogrossensis by its short bracts, and discolorous leaves. Carmen Cristóbal (1932-2019) was the first to consider this species as a new taxon.

Flowering occurs from January through mid April, June to August. Flowers may be diurnal.

Floral visitors opportunistically encountered included Editha, a male wasp (Sphecideae), wasps Pepsissp.(Pompillidae)(Fig.3A), wasps Polybiinisp. $A$, Eumenininae sp., (Vespidae), butterflies Urbanus (Hesperiidae, Lepidoptera), a moth (Noctuiidae), a fly (Tachinadae) and a hummingbird (unpubl. data, unpubl. obs. in Saunders, 1995).

Waltheria coriacea is potentially conservation assessed as of least concern (LC) by an EOO over $20,000 \mathrm{~km}^{2}$. Local reevaluation is needed. 

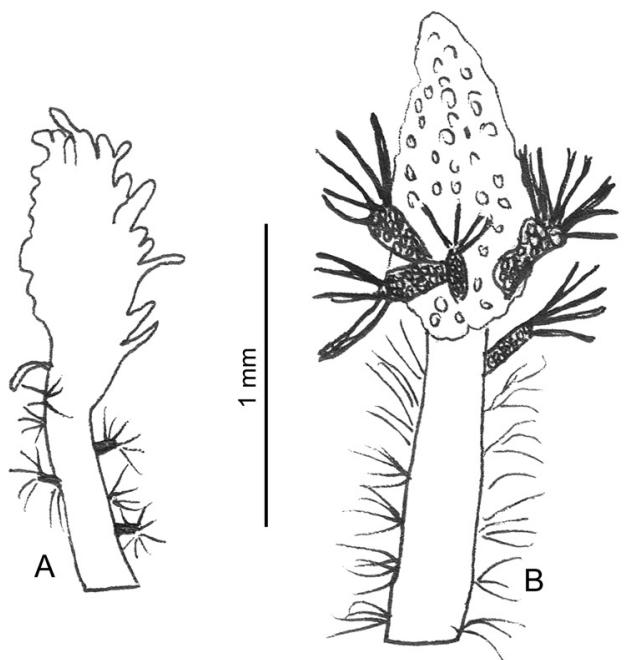

Fig. 4. Outline sketch of stipitate-stellate trichomes on style, short (A) or medium long, on style and stigma base (B). A, pin style and stigma of Waltheria coriacea. B, thrum style and stigma of $W$. matogrossensis. A, from FAA, Saunders et al. 2064 Pin. B, from FAA, Saunders et al. 3061 Thrum. Illustration: J. G. Saunders.

\section{Specimens examined.}

Paratypes. BRASIL. Bahia. Munic. de Barreiras, BR-20, $40 \mathrm{~km}$ de Barreiras, $12^{\circ} 05$ 'S, $45^{\circ} 17$ W, 26-III1984, Almeida \& Lourdes 289 (CEPEC, RB); Espigão Maestre, Serra ca. $30 \mathrm{~km} \mathrm{~W}$ of Barreiras, 3-III-1972, Anderson et al. 36517(CTES, MBM, MO, NY, R, UB, UPS); estrada para o Aeroporto de Barreiras, coletas entre 5 a $15 \mathrm{~km}$ a partir da sede do municipio, 11-VI1992, Carvalho et al. 4002 (CTES, NY, TEX); 105 km S of Barreiras, 20-II-1972, Fryxell 1920 (MICH, MO, NY); Roda Velha, 12-I-1977, Hatschbach 39440 (CTES, MBM); Rodoviaria BR-20, $30 \mathrm{~km} \mathrm{O}$ de Barreiras, 12-I-1977, Hatschbach 39479 (C, CTES, MBM, NY); Rodoviaria BR-20, 30-40 km O de Roda Velha, 20-VI-1986, Hatschbach \& Silva 50534 (CEPEC, CTES, MBM); $45 \mathrm{~km}$ E of Mimoso on BR242 Mimoso-Barreiras (48 $\mathrm{km} \mathrm{W}$ of Barreiras), $12^{\circ} 07$

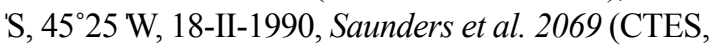
TEX, UB); $60 \mathrm{~km}$ E of Mimoso on BR-242 MimosoBarreiras, (33 km W of Barreiras), ca. $12^{\circ} 07 \mathrm{~S}, 45^{\circ} 25$ 'W, 18-II-1990, Saunders et al. 2072 (TEX, UB); 22 $\mathrm{km}$ W of Río Branco bridge on BR-242 MimosoBarreiras, $12^{\circ} 07^{\mathrm{S} S}, 45^{\circ} 15^{\mathrm{W}} \mathrm{W}, 19-\mathrm{II}-1990$, Saunders et al. 2076 (TEX, UB); $55 \mathrm{~km}$ E of Mimoso on BR-242 Brasilia-Barreiras, $33 \mathrm{Km} \mathrm{W}$ of Río Branco bridge in Barreiras, $12^{\circ} 07^{\mathrm{S} S}, 45^{\circ} 20$ W, 19-II-1990, Saunders et al. 2084 (TEX, UB); $3 \mathrm{~km} \mathrm{~W}$ of turnoff to Taguatinga de Tocantins from Mimoso (Bahia) on BR-242 (91 $\mathrm{km} \mathrm{W}$ of Barreiras de Bahia), $12^{\circ} 03^{\prime} \mathrm{S}, 45^{\circ} 40^{\prime} \mathrm{W}$, 20-II-1990, Saunders et al. 2085 (CTES, TEX, UB). Munic. Correntina: Espigão Maestre, ca. $100 \mathrm{~km}$ WSW of Barreiras, 7-III-1972, Anderson et al. 36740 (CTES, NY, UB); Espigão Maestre, Rodoviaria BR$349,64 \mathrm{~km} \mathrm{~L}$ de trevo com a Rodoviaria BR-20, 16V-2000, Hatschbach et al. 71230 (CTES). Munic. de Correntina: $40 \mathrm{~km} \mathrm{~N}$ of Possé on BR-20 Brasilia to Barreiras (198 km S of Mimoso), $13^{\circ} 55^{\prime} \mathrm{S}, 46^{\circ} 10^{\prime} \mathrm{W}$, 16-II-1990, Saunders et al. 2062 (TEX, UB); idem, Saunders et al. 2063 (CTES, TEX, UB); 46 km N of Possé on BR-20 Brasilía to Barreiras $(192 \mathrm{~km} \mathrm{~S}$ of Mimoso), 17-II-1990, Saunders et al. 2064 (TEX, UB); idem, Saunders et al. 2065 (TEX, UB); Fazenda Jatobá, entre $45^{\circ} 45^{\mathrm{E}} 46^{\circ} 15 \mathrm{~W}$, e entre $13^{\circ}$ y $14^{\circ} \mathrm{S}$, estrada da fazenda Salgada próximo ao Km 0, área de escrube, 4-III-1991, Viollati et al. 251 (CTES). Munic. Formosa do Rio Preto: Fazenda Estrondo, $79 \mathrm{~km}$ Oeste da cidade, perto do rio Riachão, $11^{\circ} 12$ '52 " S, $45^{\circ} 45$ ' 42 'W, 3-IV-2000, Oliveira et al. 467 (CTES); Coã Cerál, $10^{\circ} 33$ '58 ' S, 4541 21 'W, 28III-2000, Harley et al. 53757 (CTES). Munic. São Desidério: ca. $150 \mathrm{~km}$ SW of Barreiras, near Rio Piaú, in drainage of Río Corrente in western Bahia, 14-IV1966, Irwin et al. 14817 (F, HB, MICH, MO, NY, RB, UB); São Desidério a margen da BR-20, $13^{\circ} 46$ 'S, $46^{\circ} 09$ W, 20-III-1981, Pinto 187/81 RB (CEPEC); $142 \mathrm{~km}$ N of Possé on BR-20 Brasilía to Barreiras (96 km S of Mimoso), 17-II-1990, Saunders et al. 2066 (TEX, UB). Goiás. Munic. Guaraní de Goiás, Km 303 de BR-20, $15 \mathrm{~km} \mathrm{~N}$ do entroncamento de Possé, 16-VIII-90, Cavalcanti et al. 745 (CTES); $20 \mathrm{~km} \mathrm{~S}$ de entroncamento BR-20 y acceso a São Domingos, 19-I-1967, Cristóbal \& Krapovickas 695 (CTES). Munic. Possé, no sopé da Serra Geral de Goiás, 25-VIII-1971, I. Gottsberger \& G. Gottsberger 150-25771 (CTES). Maranhão. Tasso Fragoso, $8^{\circ} 35$ '13 'S, $45^{\circ} 49$ '53 'W, 320 m a.s.l., 7-V-2013, Santos et al. 346, (MBM); Munic. Carolina, Transamazonian Highway, BR-230, and BR-010, Pedra Caída, 35 km N of Carolina, 14-IV-1983, Taylor et al. E1210 (MO). Tocantins. Rodoviaria BR-020; $100 \mathrm{~km} \mathrm{O} \mathrm{de}$ Barreiras, 11-III-1979, Hatschbach 42067 (C, CTES, NY); Munic. Taguatinga: $13 \mathrm{~km}$ E of Taguatinga de Tocantins on road to Mimoso (Bahia) and $101 \mathrm{~km} \mathrm{~W}$ of Barreiras, Serra Espigão Maestre, $12^{\circ} 15 \mathrm{~S}, 46^{\circ} 25 \mathrm{~W}$, 20-II-1990, Saunders et al. 2087 (CTES, TEX, UB). 




Fig. 5. Range of geographic distribution of Waltheria ferruginea allies endemic to Brazil. Brazilian administrative divisions (states) with $W$. ferruginea allies: BA Bahia, CE Ceara, GO Goiás, MA Maranhão, MG Minas Gerais, MS Mato Grosso do Sul, MT Mato Grosso, PE Pernambuco, PI Piauí, TO Tocantins. Color version at http://www.ojs. darwin.edu.ar/index.php/darwiniana/article/view/928/1204

Waltheria flavovirens J.G. Saunders sp. nov. TYPE. Brazil, Goiás, $9 \mathrm{~km}$ past turnoff to Santa Rosa heading N on BR-20 to Barreiras from Brasilia, and $96 \mathrm{~km} \mathrm{~S}$ of turnoff to Flores de Goiás, $15^{\circ} 00$ 'S, $47^{\circ} 00$ W, 16-II-1990. J. G. Saunders, L. Breyer \& G. Eiten 2053 (holotype UB 217283!; isotypes CEPEC 71871!, CTES 254977!, K barcode 000381058!, MBM 209188!, TEX 00557327!). Figs. 6, 3B.

Diagnosis. Differs from $W$. brachypetala by leaves adaxial surface floccose-glabrescent, flavovirens, tomentellous hispidulous; calyx tomentose, tomentellous; petals $1.2-1.7 \mathrm{~mm}$ wide; calyx tube interior glabrous in flower, hirsute in fruit; pin pistil not geniculate at base; seed apical contour broadly 5 convex-lobed.

Shrubs 1-2 m tall. Ferruginea type stipitatestellate trichomes on vegetative parts; stipes $0.1 \mathrm{~mm}$, red, weak; stellate rays 7-13, 0.1-0.3 mm. Branches distally olive-ferrugineous, yellow tomentellous, pannose, stellate, stipitate-stellate; bark anastomose; internodes $15-50 \mathrm{~mm}$. Stipules narrowly triangular, 2.3-4.5 mm, base truncate, abaxial tomentellous, stellate, adaxial hirsutulous, stellate, glands obovoid. 
Leaves distichous, evenly distributed, reclining; petioles 6-12 × 1-3 mm, ridged; laminas concolorous yellow-green to mottled uneven color from floccose glabrescence, flat, chartaceous, variable, lanceolate or narrowly elliptic usually, elliptic, rhombic, oblanceolate, ovate, $5-16.7 \times 1.8-7.5 \mathrm{~cm}$, base rounded or subcordate for 1-3 mm usually, insertion obtuse-angled, cuneate, obtuse, apex obtuse usually, rounded, acute, margins irregularly serrate, teeth 1-4 $\times$ 0.4-0.9 mm high; adaxial surface olive to brownolive, hispidulous, tomentose-floccose, ephemeral pannose-tomentellous, stellate, some trichomes subsessile, asperous; abaxial densely tomentose, soon sparsely so, veins dark throughout, 7-10 lateral pairs above 5 basal palmate veins. Inflorescence axillary, crested, cymose, moderately dense clusters 2-8 cm, 6-32-flowered. Bract stipe 0.3-1 mm; bracts 4 per floral pair, persistent, connate at $0.5-2 \mathrm{~mm}$, 4.7-7.5 $\times 1.1-2.3 \mathrm{~mm}$, highly variable, lanceolate, obovate, entire, dentate, or irregularly 2-3 incised or praemorse, both faces densely white-tomentellous, green-pannose, stellate, abaxial also some stipitatestellate trichomes, multinerved. Bracteoles $2-4$, distinct or connate with contour obovoiddissected, very variable, irregular, 4.2-7 $\mathrm{mm}$. Flowers distylous, ca. $3 \mathrm{~mm}$ diam. corolla. Pedicels 0-0.1 mm. Calyx turbinate-clavate, strongly 10-ribbed, 5-dentate, base articulate, 4.5-6.7 $\times$ (1.8-)2.5-3.5 mm; teeth triangular-dentate, short acuminate, 1-2.5 $\mathrm{mm}$; adaxial villous, simple; interior tube glabrous in flower, hirsute in fruit, base nectary $0.2 \mathrm{~mm}$; abaxial calyx tomentose, tomentellous, stellate, stellate trichomes with basal yellow exudate. Petals 5, intense yellow, subconduplicate, oblong-obovate,(3.2-)3.6-5.7(-7) $\times$ 1.2-1.7 mm, apex retuse, eciliate or few-ciliate, two rays, adaxial surface glabrous or pilose, trichomes ca. $1 \mathrm{~mm}$, rays $1-2$, abaxial base glandular; claw $2 \mathrm{~mm}$, adnate $0.2 \mathrm{~mm}$ to stamen-base. Thrum stamens 4.5-6 mm; filaments 5, glabrous; tube 1.2$2 \mathrm{~mm}$, minutely 5-plicate at base between petals, adaxial glandular; anthers elliptic, 1-1.2 mm. Androgynophore $0.2 \mathrm{~mm}$, glandular. Thrum pistil 2.7-4.1 mm; ovary 1-1.3 mm, densely hirsute; style 0.9-1.7 mm, reclined $15^{\circ}$, stellate-hirsute to stigma or below; stigma clavate-fimbriate, lanceolate, pale green, 0.7-1.5 × 0.3-0.6 mm. Pin stamens 3-5.4 mm; filaments 5 , tube uneven, $2-4 \mathrm{~mm}$; anthers to $1.3 \mathrm{~mm}$.
Pin pistil $5.3-7.4 \mathrm{~mm}$; ovary to $1.5 \mathrm{~mm}$, basal half sparser trichomes; style 3.2-5.2 mm, twice changing direction but not geniculate at base, stellate-hirsutulous, less at apex; stigma exserted presentation excentric, clavate-fimbriate, trulloid, pale green. Capsule hidden in calyx, obovoid, 2.4$2.7 \times 1.4-2 \mathrm{~mm}$, apex obtuse angled, sericeous, puberulent, less to base, stellate, less to base to rays 2; loculicidal dehiscence incomplete; chartaceous, interior apex slightly thickened. Seed cordovan, obovoid, 1.5-2.2 × 1-1.4 mm, apex truncate-obtuse, apical contour broadly 5-convex-lobed, striate, foveate.

Etymology. The epithet refers to the "flavovirens" yellow-green color of new leaves.

Distribution and habitat. Waltheria flavovirens is endemic to Brazil, in the CW (Goiás), $\mathrm{N}$ (Tocantins), NE (Bahia) and SE (Minas Geraís), at elevations of 600-1100 m a.s.l. (Fig. 5). Occurring usually along roadsides in disturbed cerrado, cleared low woodland cerrado in Bahia, but also found in more native sites of dense hillside cerrado, in Maranhão only in cerrado s. str. (Santos 346) and also so in Goiás (Medeiros et al., 2012) on the middle Tocantins river basin, rarely in campo cerrado, or in ecotones to campo limpo, gallery margin or gallery forests in rocky, sandy yellow or red latersols with quartz pebbles, lateritic rock (toá).

Observations. Waltheria flavovirens can be distinguished from other species of $W$. ferruginea allies by its yellow-green leaves with floccose glabrescence, and seed apex apical contour broadly 5 convex-lobed.

This new species shares with $W$. biribiriensis a yellow calyx, though paler, with stellate trichomes, though smaller, paler, with an ochre substance (exudate?) at the base.

Preliminary phylogenetic analysis (Saunders, unpubl. data, discussed in Saunders, 1995) places Waltheria flavovirens between $W$. brachypetala and the sister pair clade of $W$. coriacea, $W$. matogrossensis. New characters observed indicate $W$. flavovirens shares articulate calyx bases, strong calyx ribs with close vestiture with $W$. coriacea, and adaxial petals with trichomes with two rays as a polymorphism, (petals sometimes glabrous) with $W$. matogrossensis. 


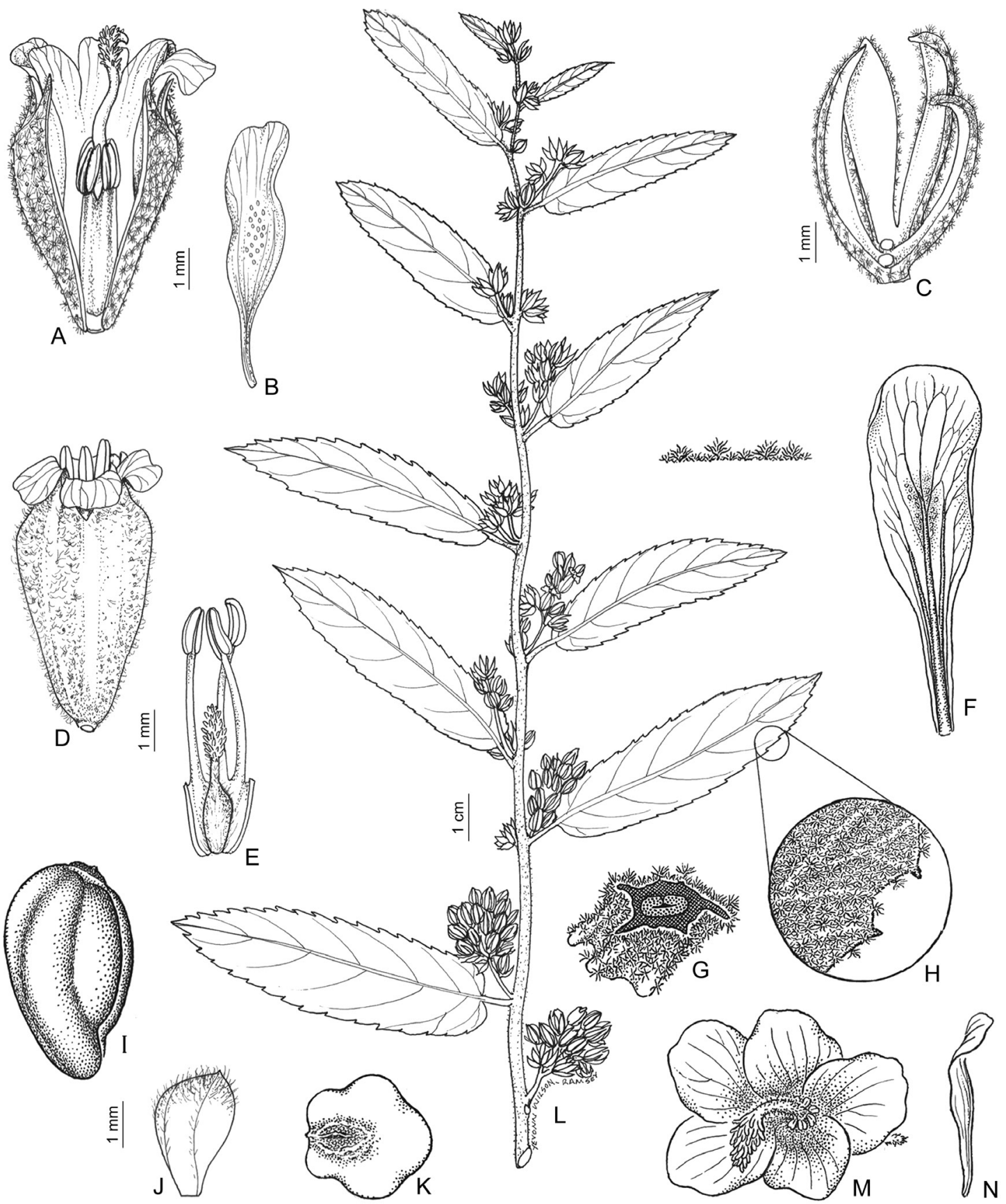

Fig. 6. Waltheria flavovirens. A, pin flower, split. B, petal, adaxial. C, two short pedicels, four adaxial bracts of floral pair. D, thrum flower. E, stamens, 3 of 5, tube split, thrum pistil. F, petal, adaxial, flattened. G, petiole. H, abaxial leaf margin. I, seed, lateral view. J, capsule. K, seed, apical view. L, flowering branch and detail, adaxial leaf vestiture, tomentose overlayer, pannose-tomentellous underlayer. M, pin flower. N, subconduplicate petal, natural form. A-F, L from Saunders et al. 2055; G-H from Saunders et al. 2056; I-K from Saunders et al. 3024-3025 (CTES); M-N from FAA, Saunders et al. 2053-2057. Illustration: Figs. A-E, J, L by Y. Wilson-Ramsey; Figs. F-I, K, M-N later by F. Rojas. 
Flowering occurs from November through July, peaking in February and March. Flowers may be diurnal.

Opportunistic observations indicate that Trigona $s p$. bees (Apidae) are likely one of the effective pollinators, contacting both floral organs. Other visitors were Pepsis $s p$. wasp (Pompilidae), a medium sized black Vespidae wasp, Editha sp. (Sphecidae), honeybees, and flies (Syrphidae, Tachinidae) (Saunders, unpubl. data, obs. in Saunders, 1995). Burchell 86322 is a voucher for Köhler (1976) and Moulton (1908). Moulton reports "Cystineura tocantina Bates \# 1316-663 (Rhopalocera, Lepidoptera) feeding on flowers of Burchell 8632-2. The lepidopteran is reported from the Porto Real (Nacionale) collected 17-II-1829."

One remnant natural population had 3 thrums: 2 pins plant ratio (Saunders et al. 2055), others were adventive, anisoplethic.

Plants regenerate from a ground level bole after fires.

Waltheria flavovirens is potentially conservation assessed as of Least Concern (LC), having an EOO over $20,000 \mathrm{~km}^{2}$. Regions around Niquelândia have nickeliferous and serpentine soils, many endemic grasses, and are a conservation priority area (Filgueiras, 2002: 124).

\section{Specimens examined.}

Paratypes. BRAZIL. Bahia. Munic. Correntina: Rodovia Brasilia-Fortaleza, entre o rio Correntes e Sta. María, 4-VII-1964, Pires 58156 (CTES, F, NY, S, UB, US). Goiás. Munic. Agua Fría de Goiás: $7 \mathrm{~km}$ by road S of São João da Aliança, 22-III-1973, Anderson et al. 7674 (CTES, NY, UB); Rodoviaria GO-118, subida para a Torre Repetidoro de Roncado, s.d., without collector s.n. (CTES 320129). Munic. Alto Paraíso de Goiás: Chapada dos Veadeiros, $12 \mathrm{~km}$ E de Colinas, 04II-1990, Arbo et al. 3663 (CTES). Munic. Campo Belos: estrada de Chão Oara Pouso Alto, $12^{\circ} 59$ '54 " S, $46^{\circ} 25$ '50 " W, 24-IV-2001, Fonseca et al. 2578 (CTES). Munic. Cavalcante: Chapada dos Veadeiros, ca. $7 \mathrm{~km} \mathrm{~S}$ of Cavalcante, 8-III-1969, Irwin et al 24021 (CTES, INPA, NY, SPF, UB); ca. $3 \mathrm{~km}$ S of São João da Aliança, $850 \mathrm{~m}$ a.s.1., 16-III1971, Irwin et al. 31951 (CTES, MO, NY, UPS). Munic. Colinas do Sul: alrededores de Colinas, sobre el camino a Cavalcante, 05-II-1990, Arbo et al. 3693 (CTES, TEX). Munic. Flores de Goiás, 44,4 km da BR-020 para Flores de Goiás, 16-I1997, Werneck et al. 927 (CTES). Munic. Formoso: BR-20, 26 km NE of Formosa, 02-II-1990, Arbo et al. 3480 (CTES, TEX); Region of Southern Serra Dourada, $23 \mathrm{~km}$ E of Formoso, $13^{\circ} 45 \mathrm{~S}, 48^{\circ} 50$ 'W, 21-V-1956, Dawson 15037 (MO, US); Vale do Paraná, Duarte 10306 (CTES, R not seen); Vale do Paraná, 3 k, 5-II-1967, Duarte 10306 (CTES, R not seen). Munic. Monte Alegre de Goiás: 3 km SW of Monte Alegre de Goiás on road GO-118 to Brasilia D.F., $13^{\circ} 10$ 'S, $47^{\circ} 05^{\prime} \mathrm{W}, 21-\mathrm{II}-1990$, Saunders et al. 3001 (CTES, TEX, UB); $2 \mathrm{~km}$ SW of Monte Alegre from center of Monte Alegre de Goiás on road (GO118) to Brasilia D.F., $13^{\circ} 10$ ', S $47^{\circ} 05$ W, 21-II1990, Saunders et al. 3010 (CTES, TEX, UB); 28.5 $\mathrm{km} \mathrm{S}$ of Monte Alegre de Goiás on road (GO-118) to Brasilia D.F., $13^{\circ} 20$ 'S, $47^{\circ} 10$ 'W, 22-II-1990, Saunders et al. 3013 (CTES, TEX, UB); idem, Saunders et al. 3014 (CTES, TEX, UB); 24 km by road SW of Monte Alegre de Goiás, 11-III-1973, Anderson et al. 6791 (CTES, MO, MBM, NY, UB, UPS). Munic. Niquelândia, estrada paralela ao Río Tocantins, entre o Río Tocantins e Serra Negra, $13^{\circ}$ $59 \mathrm{~S}, 41^{\circ} 21 \mathrm{~W}, 25-\mathrm{VII}-95$, Cavalcanti et al. 1674 (CTES); ca. 25 km S of Niquelândia, 24-II-1972, Irwin et al. 34940 (CTES, NY, TEX); $37 \mathrm{~km} \mathrm{~N}$ of Dois Irmãos de Goiás on road to Niquelândia from Padre Bernardo, ca. $14^{\circ} 50$ 'S, $48^{\circ} 38^{\prime}$ W, 27-II-1990, Saunders et al. 3037 (CTES, TEX, UB); idem, Saunders et al. 3039 (CTES, TEX, UB); $82 \mathrm{~km} \mathrm{~N}$ of Dois Irmãos de Goiás on road to Niquelândia from Padre Bernardo, ca. $14^{\circ} 30$ 'S, $48^{\circ} 30^{\prime} \mathrm{W}, 27-\mathrm{II}-1990$, Saunders et al. 3042 (CTES, TEX, UB); idem, Saunders et al. 3043 (TEX, UB); 55 km S of Faz Tudo on road between Dois Irmãos and Niquelândia from Padre Bernardo, ca. $14^{\circ} 30^{\prime} \mathrm{S}, 48^{\circ} 30^{\prime} \mathrm{W}, 27-\mathrm{II}-$ 1990, Saunders et al. 3044 (CTES, TEX, UB); 58 $\mathrm{km} \mathrm{S}$ of Faz Tudo on road between Dois Irmaos and Niquelândia, 27-II-1990, Saunders et al. 3045 (UB); $59 \mathrm{~km} \mathrm{~S}$ of Faz Tudo on road between Dois Irmaos and Niquelândia, 27-II-1990, Saunders et al. 3046 (CTES, TEX, UB); idem, 27-II-1990, Saunders et al. 3047 (TEX, UB). Munic. São João d'Aliança: Chapado dos Veadeiros región, $19 \mathrm{~km} \mathrm{~N}$ of São João da Aliança, Ribeirão Capetinga, ca. $14^{\circ} 30$ 'S, $47^{\circ} 30 \mathrm{~W}, 17-\mathrm{IV}-1956$, Dawson 14368 (US); $3 \mathrm{~km} \mathrm{~S}$ of São Jão da Aliança de Goiás on road to Brasilia 
D.F. ca. $14^{\circ} 40$ 'S, $47^{\circ} 35$ 'W, 23-II-1990, Saunders et al. 3024 (CTES, TEX, UB); idem, Saunders et al. 3025 (CTES, TEX, UB); Córrego das Brancas, proximo a barra do Jacaré, 9-II-1994, Hatschbach et al. 60182 (CTES). Munic. Uruaçu: Uruaçu, arredores, 30-I-1976, Hatschbach \& Ramamoorthy 38214 (C, CTES, MBM, US); Munic. Água Fria: Córrego Ribeirão, 7-XI-1991, Hatschbach \& Silva 55878 (CTES); BR-153, Uruaçu, km 256, $14^{\circ} 30$ S, $49^{\circ} 8^{\prime}$ W, 3-III-1982, Krapovickas et al. 37816 (CTES). Munic. Vila Boa: $9 \mathrm{~km}$ past turnoff to Santa Rosa heading N on BR-20 to Barreiras from Brasilia, and $96 \mathrm{~km} \mathrm{~S}$ of turnoff to Flores de Goiás, $15^{\circ} \mathrm{S}, 47^{\circ} \mathrm{W}, 16-\mathrm{II}-1990$, Saunders et al. 2054 (CTES, TEX, UB); idem, Saunders et al. 2055 (TEX, UB); idem, Saunders et al. 2056 (TEX, UB); idem, Saunders et al. 2057 (TEX, UB). Munic. Vila Propicio: $5 \mathrm{~km}$ N of Dois Irmaos de Goiás on road to Niquelandia from Padre Bernardo, ca. $15^{\circ} \mathrm{S}, 48^{\circ}$ 40 W, 27-II-1990, Saunders et al. 3036 (CTES, TEX, UB). Minas Gerais. Munic. Bocaiúva, Rodoviaria Bocaiúva-Diamantina, próximo ão Rio Jequitinhonha, 17-III-1997, Hatschbach et al. 66427 (CTES, MBM). Munic. Brasópolis: Brazilândia de Minas, Fazenda Brijão $16^{\circ} 58^{\prime} 43^{\prime \prime} \mathrm{S}$, $45^{\circ} 47^{\prime} 14$ 'W, 13-VII-2000, Lombardi 3980 (BHCB, CTES). Munic. Buenopolis, Curimataí, $40 \mathrm{~km}$ E of BR-135, entre Buenopolis y Joaquim Felicio, $17^{\circ} 51$ ' S, 43 $58^{\prime}$ W, 21-V-1990, Arbo et al. 4552 (CTES, TEX). Munic. Buritizeiro, Rodoviaria BR-365, descida da Chapada dos Gerais, 11-III-1995, G. \& M. Hatschbach 62101 (CTES, MBM). Munic. de Formosa, Parque Nacional Grande Sertão Veredas, na margen do Riberirão Mato Grande, cerca de $1 \mathrm{~km}$ da cachoeira próximo à Fazenda do Sr. Nenzinho, $15^{\circ} 1933^{\prime \prime} \mathrm{S}, 45^{\circ} 59$ '17 " W, 16-II-1999, Mendonça et al. 3783 (CTES, IBGE). Munic. João Pinheiro: além de João Pinheiro, margen de rodovia, 16-II1972, Heringer 12050 (UB); João-Pinheiro, VIII1998, Brina s.n. (BHCB-49302, CTES). Munic. Varzea da Palma, Fazenda Mãe D’Agua, 26-IV1963, Duarte 7844 (NY, RB); idem, 1992, Duarte 9888 (CTES). Tocantins. Munic. Porto Nacional: Porto Reál, Nacionale, 17-II-1829, Burchell 86322 (K). State unknown. Burchell 8072 (K); Pohl 920 (PR); in via a Pilar [Pilar de Goias?] ao Faz. Lavrinhas, Pohl 1676, Herb. Vien no. 1329 (K, W); Serra do Allegres [Goias?], 1837, Pohl 3359, Herb. Mus. Vind. no. 1357 (GH, K, W).
Waltheria hatschbachii J.G. Saunders, sp. nov. TYPE. Brazil. Minas Gerais: Munic. Cunha Magalhães, Mendanha, 16-XI-1971, G. Hatschbach \& P. Pelanda 28038 (holotype MBM 19756! [thrum]; isotypes C barcode 10019406! [thrum], CTES 35173! [thrum], US 2700775, barcode 00479016!). Fig. 7.

Diagnosis. Differs from $W$. brachypetala by leaf margins crenulodentate, adaxial surfaces not visible; inflorescence 2-8-flowered; bracts narrowly triangular, curved; petal adaxial hairs arachnoid at midlength, simple; thrum stamens $6.3 \mathrm{~mm}$, stamen tube 1.4-1.8 $\mathrm{mm}$; thrum androgynophore woody; thrum pistil 5.3-5.8 $\mathrm{mm}$.

Shrubs 1-2.5 m tall. Ferruginea type stipitatestellate trichomes on vegetative parts and calyx; stipe ochre to red, ca. $0.1-0.3 \mathrm{~mm}$, stellate subglobose rays ca. 30 , ca. $0.2 \mathrm{~mm}$, white to yellowish ochre based. Branches distally cinereoustomentellous, minute pannose, stellate, numerous stipitate-stellate; bark resinous, castaneous, black, striate; internodes 2-20 mm, nodes knobby; stem interior dark brown. Stipules narrowly triangular, linear triangular, canaliculate, $2-10.3 \mathrm{~mm}$, base truncate, abaxial tomentellous, stellate, stipitatestellate, adaxial stellate, glands pyriform. Leaves distichously arranged, closely spaced at apices, horizontal; petioles 2-7 $\times$ 0.7-1.4 mm, ridged; laminas concolorous, surface not visible, flat, firm, lanceolate, at timesfalcate, $1.3-9.3 \times 0.4-3 \mathrm{~cm}$, apex obtuse, acute, apiculate, base rounded, subcordate for 2-4 $\mathrm{mm}$, insertion planar, margins crenulodentate, teeth $1-2.5 \times 0.3-1 \mathrm{~mm}$, adaxial surface ferrugineous-olive, whitish tomentose, tomentulose, dense stipitate-stellate, slightly asperous; abaxial olive, pale incanus (whitish), tomentose, tomentellous, dense stipitate-stellate, veins 7-11 lateral pairs above 9 palmate basal veins, $1^{\circ}-5^{\circ}$ order veins raised. Inflorescence axillary, crested, cymose, few-flowered clusters $0.8-1.5 \mathrm{~cm}$, ca. 2-8-flowered. Bract stipe $0.2 \mathrm{~mm}$; bracts 4 per floral pair, persistent, connate at $0.4-0.9 \mathrm{~mm}, 6.2-$ $10 \times 0.7-1.8 \mathrm{~mm}$, narrowly triangular, acute, entire, curved, tomentellous, stellate, stipitate-stellate. Bracteoles 3, 2-3-connate, linear triangular, 4$4.5 \mathrm{~mm}$. Pedicels $0-0.4 \mathrm{~mm}$. Flowers presumably distylous, pins unknown, ca. $3 \mathrm{~mm}$ diam. corolla. 


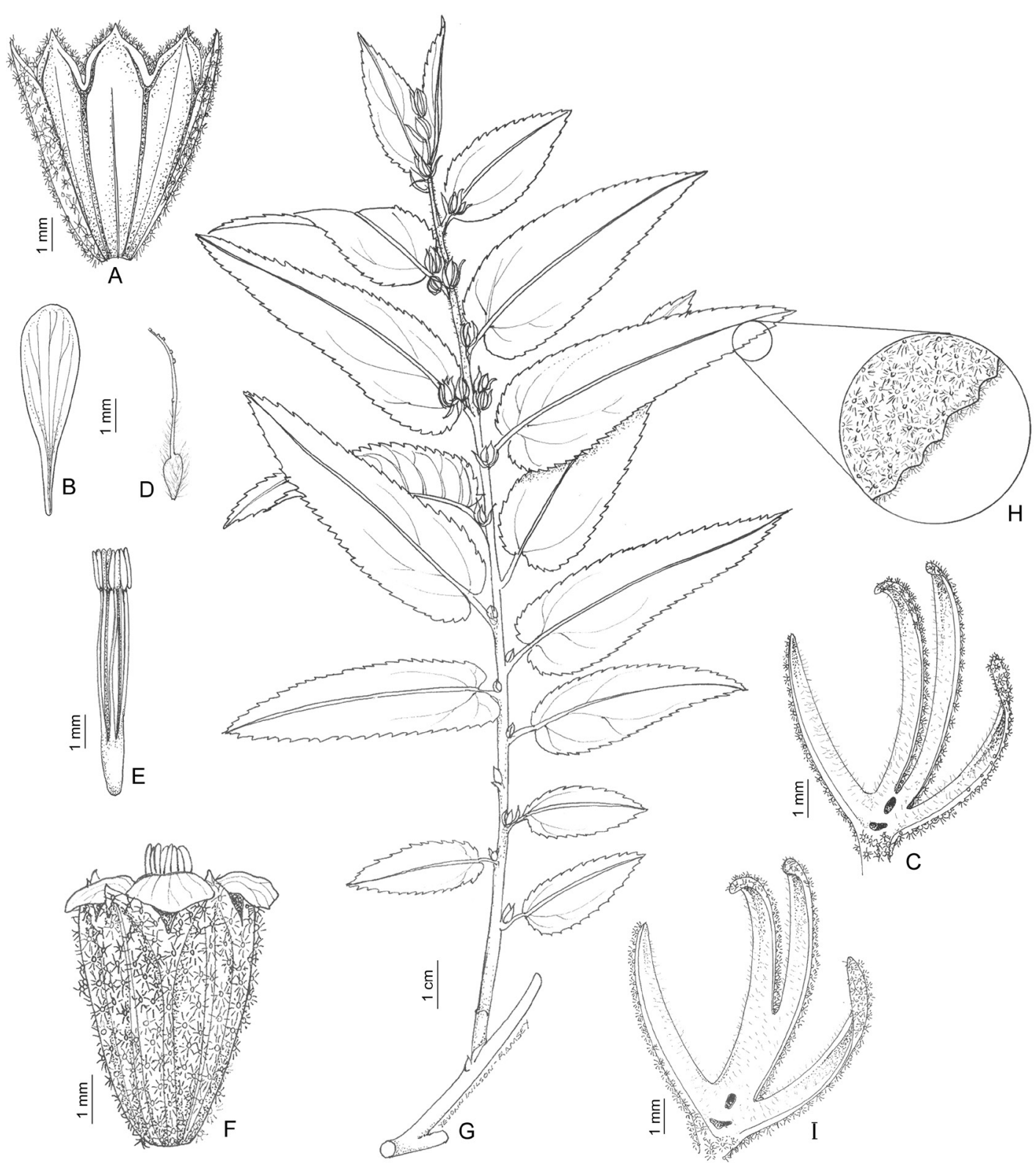

Fig. 7. Waltheria hatschbachii. A, calyx split, adaxial. B, petal, adaxial flattened. C, two sessile flower scars, four adaxial bracts of floral pair. D, thrum pistil. E, thrum stamens. F, thrum flower. G, flowering branch. H, leaf margin, adaxial. I, two sessile flower scars, four adaxial bracts of floral pair. All from Hatschbach \& Pelanda 28038 (MBM). Illustration: Y. Wilson-Ramsey.

Calyx turbinate-clavate, 5-angled, 5-ribbed, 5-dentate, $6.5-7 \times 3.5-4.5 \mathrm{~mm}$; teeth triangularovate-mucronate, $1.5-2 \times 1.3-1.7 \mathrm{~mm}$, interior villulose, trichomes simple; tube interior villulose, puberulent, base nectary to $0.8 \mathrm{~mm}$; exterior calyx tomentellous, globose-stellate, yellow to ochre, some trichomes stipitate-stellate. Petals 5, yellow, spathulate, subconduplicate, oblanceolate, 6-6.1 $\times$ 1.5-2 mm, apex rounded, eciliate, adaxial surface arachnoid simple, abaxial glabrous, base thick, triangular, dilated; claw $1 \mathrm{~mm}$, adherent $0.1 \mathrm{~mm}$ to stamen-base. Thrum stamens $6.3 \mathrm{~mm}$, papillose; 
filaments 5, glabrous, tube 1.4-1.8 mm; anthers 1.1-1.2 mm. Thrum pistil 5.3-5.8 mm; ovary 1.1$1.3 \mathrm{~mm}$, densely velutinous sericeous to base; style 2.3-2.7 mm, velutinous, stellate, sparsely so on distal half, not stipitate; stigma curved, clavatefimbriate, oblong (Lombardi 3811) to flat, lineartriangular, papillose (Hatschbach 28038), 1.2-1.5 $\times$ $0.2-0.5 \mathrm{~mm}$. Androgynophore $0.2 \mathrm{~mm}$ in flower, chartaceous, in fruit to $0.7 \mathrm{~mm}$, woody, glandular, with calyx base adpressed. Capsule hidden in calyx, oblong-ovoid, 2.7-1.5 mm, apex rounded, hirsutulous, tomentose, less to base, stellate, less rays to base where rays 2 ; loculicidal dehiscence incomplete, in apical third; chartaceous, interior apex very slightly sclerified. Seed castaneous, oblong-ovoid, 1.9-2.2 × $1.3 \mathrm{~mm}$, apex rounded, minutely verrucose, foveate, papillose; chalaza 0.1-0.3 mm high.

Etymology. The species name honors the distinguished Brazilian botanist Gert Hatschbach $\dagger$, founder and former director of Museu Botânico Municipal at Curitiba, Brazil, whose life's work helped to unveil the incredible diversity in the Brazilian flora.

Distribution and habitat. Waltheria hatschbachii is endemic to SE Brazil in Minas Gerais, from two vicinities near Diamantina: Mendanha, about $30 \mathrm{~km}$ NE of Diamantina, and $52 \mathrm{Km}$ NE of Diamantina in the State Park São Gonçalo do Rio Preto (Fig. 5). It occurs in cerrado at 900-1200 m a.s.1., in sandstone outcrops or sandy soil.

Observations. Waltheria hatschbachii can be distinguished by its bracts long, triangular, at times curved about $6-10 \mathrm{~mm}$, finely tomentellous lanceolate leaves, often falcate, always subcordate or cordate, margins crenulodentate, and leaf lamina bases often overlapping due to stem short internodes $2-20 \mathrm{~mm}$. Unique to $W$. hatschbachii are the arachnoid simple trichomes on adaxial petals, and woody glandular (reduced) androgynophore below thrum pistils. The petals are very resinous, lateral margins ruffled, unusual in the $W$. ferruginea alliance. Thrum pistils are the longest in the W. ferruginea allies but ones nearly as long were found in $W$. terminans $(3.2-5.2 \mathrm{~mm})$ and $W$. biribiriensis $(2.4-5 \mathrm{~mm})$.
Waltheria hatschbachii shares with $W$. biribiriensis ferruginea type stipitate-stellate trichomes with multiradiate rays ca. 30, as in those two species and $W$. brachypetala. Some examples of the type of $W$. hatschbachii have thrum stigmas curved and few papillose as in $W$. biribiriensis, other examples (Lombardi 3811) have stigmatic branches 0.1-0.2 $\mathrm{mm}$, and are clavate-fimbriate. Waltheria hatschbachii shares subcordate, cordate leaf bases, at times with bases overlapped between distichous, sequential leaves along the flowering branch, with $W$. biribiriensis, the latter species with longer internodes, 8-35 $\mathrm{mm}$.

Some of the previous material has been identified as $W$. brachypetala. It can be distinguished from $W$. brachypetala by its bracts narrowly triangular, 6-10 $\times 0.7-1.8 \mathrm{~mm}$ (vs. obovate, elliptic, $4.5-7.5 \times 1-3.8 \mathrm{~mm}$ ) by the absence of the thin white stellate-velutinous overlayer on the bracts and calyx, and by its thrum pistil 5.3-5.8 $\mathrm{mm}$ (vs. 3.5-4.3 $\mathrm{mm}$ ).

Flowering in April, October, November.

Waltheria biribiriensis, at 800-1234 m a.s.1., and $W$. terminans, at 1100-1300 m a.s.l., both with some populations also occurring along a 30 Km radius NE of Diamantina to Mendanha, by mapped localities appear to be sympatric, but by their occurrence in campo rupestre rather than in cerrado given for this species (Hatschbach \& Pelanda 28038; Lombardi 3811, 4181) appear to be ecologically distinct.

Waltheria hatschbachii needs local conservation assessment but could be potentially classified now as Endangered (EN) by B1 geographical extent of occurrence $(\mathrm{EOO})<5,000$ $\mathrm{km}^{2}$ [with an estimated $42 \mathrm{~km}$ transect length] and B2a by 3 or possibly actually 2 localities known, thus $<5$, and B2b by urban development causing a decline of (iv) subpopulation number by loss of the Mendanha population, if it exists there, or (v) loss in number of individuals.

\section{Specimens examined.}

Paratypes. BRAZIL. Minas Gerais. Munic. Felisberto Caldeira: Parque Estadual do Rio Preto, São Gonçalo do Rio Preto-MG da casa de hóspedes ( $\left.18^{\circ} 07^{\prime} 34^{\prime} \mathrm{S}, 43^{\circ} 21^{\prime} 24^{\prime \prime} \mathrm{W}\right)$ a cascata do

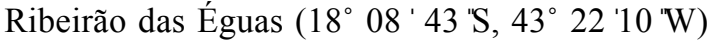


e então por fim ao camping ( $18^{\circ} 06^{\prime} 54^{\prime} \mathrm{S}, 43^{\circ} 20$ 28 "W), 08-IV-2000, Lombardi et al. 3811 (BHCB51438 not seen, CEPEC, CTES); São Gonçalo do Rio Preto-MG, Parque Estadual do Río Preto, trilha para a cascata do Córrego das Éguas, daí seguindo o curso até Rio Preto eo camping, entre $18^{\circ} 08^{\prime} 43^{\prime} \mathrm{S}, 18^{\circ} 06^{\prime} 54^{\prime \prime S}$ y entre $43^{\circ} 22^{\prime} 10^{\prime} \mathrm{W}$, $43^{\circ} 2028 \mathrm{~W}, 19-\mathrm{X}-2000$, Lombardi 4181 (BHCB 53896 not seen, CTES thrum).

Waltheria hoehnei J.G. Saunders, sp. nov. TYPE. Brazil, Mato Grosso, Munic. Cuyabá [Cuiabá], Coxipó da Ponte, IV-1911 F. C. Hoehne 3032 (holotype SP 55193! [pin]). Figs. 8, 9.

Diagnosis. Differs from $W$. brachypetala by leaf surfaces concolorous, adaxial surfaces not visible; bracts 2.4-3.5 × 0.6-0.9 mm; corolla $5 \mathrm{~mm}$ diam.; pin pistil $8.9 \mathrm{~mm}$; capsule compressed, apex inclined-truncate; seed rugulose, verrucate, thick (ca. $0.2 \mathrm{~mm}$ ) walled, chalaza crenate.

Shrubs to $1 \mathrm{~m}$ tall. Ferruginea type stipitatestellate trichome on vegetative parts; stipe 0.1$0.2 \mathrm{~mm}$, ochre, red, breaking; stellate rays ca. $12-20,0.2-0.3 \mathrm{~mm}$, yellowish. Branches distally densely tomentose, pannose, stellate, stipitatestellate, glabrescent; bark resinous, castaneous, striate; internodes 12-20 mm. Stipules narrowly triangular, 2.7-6 $\times$ 0.3-0.6 mm, abaxial densely stellate tomentose, some stipitate-stellate trichomes, adaxial same, simple, stellate, glands obovoid, pyriform under dense white setae. Leaves distichous, evenly distributed close together, horizontal; petioles 3-5 $\times 1-2 \mathrm{~mm}$, ridged; laminas concolorous, surface not visible, flat, oblong-lanceolate usually, lanceolate, ovate, oblong-ovate, elliptic, $6-9 \times 2.8-4.3 \mathrm{~cm}$, base rounded, insertion obtuse-angled, apex acute, margins serrulate, teeth 1.7-3.5 $\times 0.4-1.2 \mathrm{~mm}$, olive green, very soft, tomentellous, pannose, stellate, stipitate-stellate, abaxial surface same but densely stipitate-stellate, veins 9 lateral pairs above 5 basal palmate veins. Inflorescence axillary, crested, cymose, loose triangular clusters 1-4.5 cm, 4-80-flowered. Bract stipe $2 \mathrm{~mm}$; bracts 4 per floral pair, distinct, dark, short, 2.4-3.5 $\times$
0.6-0.9 mm, triangular, narrowly ovate, elliptic, acute, all entire, adaxial pubescent, sericeous, simple, rays $2,0.2 \mathrm{~mm}$, glands pyriform, abaxial as stem. Bracteoles 2-3, connate or distinct, 1.7$2 \times 0.4-0.6 \mathrm{~mm}$, narrowly triangular. Flowers sessile, presumably distylous, thrums unknown, ca. $5 \mathrm{~mm}$ diam. corolla. Calyx turbinate-clavate, 5-ribbed, 5-plicate, 5-dentate, 6-6.5 × 3-4 mm, teeth ovate-deltate-mucronate, $1-1.6 \mathrm{~mm}$, interior villulose, sericeous, trichomes simple, or rays $\geq 2$; tube interior sericeous, base nectary $0.7 \mathrm{~mm}$; calyx exterior tomentellous, stellate, stipitatestellate. Petals 5, dark orange below a pale yellow rim (dried), spathulate, subconduplicate, flat apex, obtriangular, 6-6.3 × 2.4-2.5 mm, truncate-retuse, eciliate, adaxial surface with sessile glands, abaxial surface stellate-pubescent, minute, few short trichomes, simple or two rays; claw $2 \mathrm{~mm}$, adherent $0.2 \mathrm{~mm}$ to stamen base. Pin stamens $5.3 \mathrm{~mm}$, filaments 5 , papillose, unevenly connate, tube $3.5 \mathrm{~mm}$; anthers $1.2-1.3 \mathrm{~mm}$, divergent empty, thecae unlevel, free $0.3 \mathrm{~mm}$ at apex and base. Pin pistil $8.9 \mathrm{~mm}$, dark, red papillose, long exserted excentric presentation of stigma; ovary $1.3 \mathrm{~mm}$, densely canescent, velutinous, stellate, glabrous portion null; style long-exserted, presentation with stigma excentric, $6 \mathrm{~mm}$, sparsely pubescent, hirsutulous, stellate, few trichomes in apex; stigma angled to $45^{\circ}$, clavate-fimbriate, ellipsoid, 1.3-1.6 $\times 0.6-0.8 \mathrm{~mm}$, a stellate trichome at base, not stipitate-stellate. Capsule hidden in calyx, very compressed, dimidiate-obovate, 3.5-3.8 $\times$ 1.9-2 mm, apex inclined-truncate, hirsutulous, tomentose, stellate, glabrous, fewer rays to 2 toward base, papillose; loculicidal dehiscence incomplete; chartaceous exterior at apex and base, membranous in $2 / 3$ length, apex adaxialy slightly sclerified. Seed unexpanded, plicate, obliquely obtrulloid, $2 \times 1 \mathrm{~mm}$, rugulose, densely verrucate (Fig. 10), red papillose, apex with oblong ridge; chalaza calyptrate, crenate; seed wall very thick, ca. $0.2 \mathrm{~mm}$, dark, very hard.

Etymology. Waltheria hoehnei commemorates the Brazilian botanist Federico C. Hoehne (1882-1959), founder of the Instituto Botânica de São Paulo, who collected the type and wrote a book about medicinal or toxic Brazilian plants, including medicinal uses of Waltheria (Hoehne, 1939: 191-193). 




Fig. 8. Waltheria hoehnei. A, calyx split, adaxial. B, petal, adaxial flattened. C, floral pair, two of four bracts, opposed by three bracteoles. D, pin pistil. E, pin stamens. F, pin flower. G, flowering branch. H, leaf margin, abaxial. I, capsule. All from Hoehne 3032 (SP-55193). Illustration: Y. Wilson-Ramsey.

Distribution and habitat. Waltheria hoehnei is endemic to Mato Grosso in CW Brazil, restricted to two municipalities SE of Cuiabá: Munic. Coxipó da Ponte and Munic. Jacimeira, Balneário Áquas Quentes (Fig. 5). It occurs at ca. $360 \mathrm{~m}$ s.a.l. in "capão do cerrado junto de lajedo com presença de mandacarús
(Cactaceae)... margen de mata" (PietrobomSilva 3574). Flowering in April and October.

Observations. Hoehne appears on the left margin label as botanist on the photocopy of the holotype specimen, and Kuhlman originally determined it to $W$. ferruginea . 




Fig. 9. Waltheria hoehnei, paratype, Hoehne 3028 (R). Photo by Nilber Gonçalves de Silva, used with permission, segment composed by R. Donadio. Color version at http://www.ojs.darwin.edu.ar/index.php/darwiniana/article/view/928/1204 




Fig. 10. Seed of Waltheria hoehnei. From Hoehne 3032 (SP-55193). Color version at http://www.ojs.darwin. edu.ar/index.php/darwiniana/article/view/928/1204

Waltheria hoehnei is distinguished by its showy dark orange pale yellow rimmed (dry) corolla ca. $5 \mathrm{~mm}$ diam. vs 3-4 diam in the rest of the allies, short bracts, shorter even than the 3-4.5 $\mathrm{mm}$ bracts found in $W$. coriacea, and for the very long pin pistil, $(8.9 \mathrm{~mm})$, others in the allied species usually to $7.5 \mathrm{~mm}$ or in $W$. brachypetala, to $5.8-8 \mathrm{~mm}$. Its anthers, with divergent thecae when empty, and non-level between thick bases or apices, one higher, are different, seen in only a few species (e.g. $W$. fryxellii J.G. Saunders).

The rugulose, verrucose seed, very thick walled, and chalaza crenate is almost unique within the genus (Fig. 10), although sect. Stegowaltheria has subtuberculate, verrucate seeds.

Waltheria hoehnei is potentially conservation assessed as Endangered (EN) by B1 geographical extent of occurrence (EOO) $<5,000 \quad \mathrm{~km}^{2}$ [estimated transect length $140 \mathrm{~km}$ ], and $\mathrm{B} 2 \mathrm{a}$ number of localities $\leq 5$ [two known, one not since 1911] and B2b decline in habitat by urban development projected in (iii) extent of habitat or (iv) number of localities. If the type locality population no longer exists, it would become potentially reclassified as Critically Endangered
(CR) $\mathrm{B} 1$ by $\mathrm{EOO}<100 \mathrm{~km}^{2}$ and $\mathrm{B} 2 \mathrm{a}(\mathrm{AOC})$ and $\mathrm{b}$ projected decline by urban development in (i) EOO, (iii) AOO or (iv) decline in locality number.

\section{Specimens examined.}

Paratypes. BRAZIL. Mato Grosso. Munic. Cuyabá [Cuiabá], Coxipó da Ponte, IV-1911, Hoehne 3027 (R pin), idem Hoehne 3028 (R pin); idem Hoehne 3029 (R pin); idem Hoehne 3030 (R pin); idem Hoehne 3031 (R); Munic. Jucimeira [Juscimeira?]: Balneário Áquas Quentes, ca. $15^{\circ} 48 \mathrm{~S}, 55^{\circ} 25^{\mathrm{K}} \mathrm{W}$, ca. $12 \mathrm{~km}$ da BR-364, 04-X-1996, Pietrobom-Silva et al. 3574 (CTES pin).

Waltheria matogrossensis J.G. Saunders, sp. nov. TYPE. Brazil, Mato Grosso, Munic. Cuiabá, Chapada dos Guimarães, Morro São Jerônimo, entre a Rodovia e o Morro, $4 \mathrm{~km}$ depois da Casa de Pedras, 25-VI1990, N. Saddi 12445 (holotype: UFMT! [pin]; isotypes: CTES 254973! [pin], TEX 00557328! [pin]). Figs. 11, 3C, 4B.

Diagnosis. Differs from $W$. brachypetala by stem vestiture tomentose-woolly with stipitate-stellate trichomes rays 7-12, stipes $0.06-0.5 \mathrm{~mm}$; leaf laminas elliptic, ovate, $4-10 \mathrm{~cm}$ wide, apex obtuse usually, tomentose-velutinous, surface not visible when young; bracts subequal, narrowly lanceolate, apex entire, short glandular trichomes on both sides; petal margin always emarginate, sinus ca. $0.3 \mathrm{~mm}$, adaxial surface scant pilose, hairs ca. 10, simple, rays two, 0.5-0.7 mm; adaxial pin staminal tube setose, hairs pointing to base; pin style densely stellate base to stigma, short stiped stipitate-stellate hairs at stigma base and style apex.

Shrubs 0.5-3 m tall, without main trunk, branches arcuate. Ferruginea type stipitatestellate trichomes present on vegetative parts and abaxial calyx; stipes red, weak, ca. 0.06-0.5 mm; stellate rays ca. 7-12, ca. 0.4-0.7 mm. Branches distally yellowish, ferrugineous-dotted, densely tomentose-woolly, cinereus-tomentose-pannose, stellate, stipitate-stellate; bark cordovan, grayish, ridges anastomose; internodes 25-80 $\mathrm{mm}$. Stipules narrowly triangular, 1.3-3.5 $\mathrm{mm}$, 


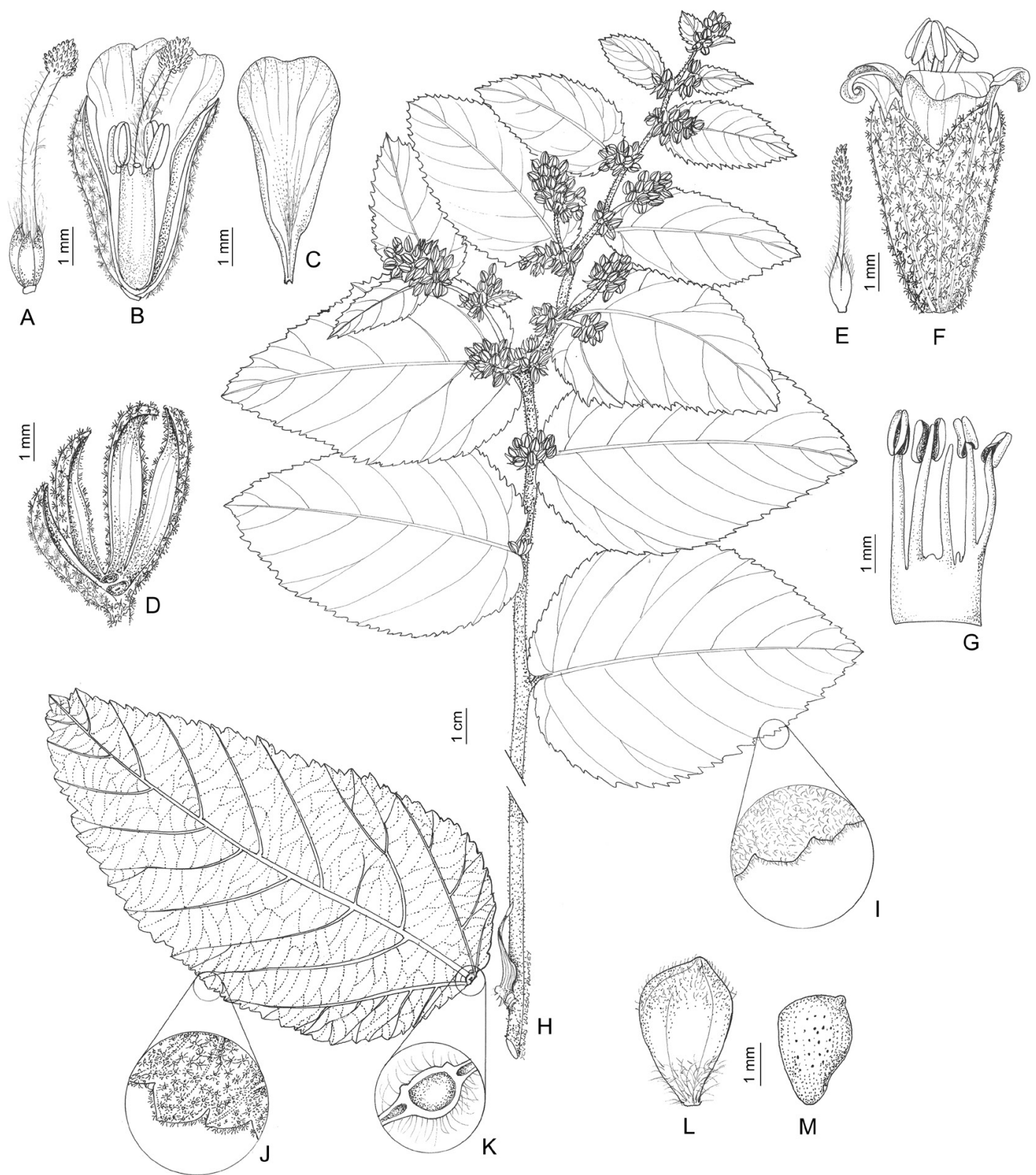

Fig. 11. Waltheria matogrossensis. A, pin pistil, broken. B, pin flower, split. C, petal, adaxial. D, two flower scars, four adaxial bracts of floral pair. E, thrum pistil. F, thrum flower. G, stamen tube opened, adaxial, with lobules of vestigial extra stamens. H, flowering branch. I, leaf margin, adaxial. J, leaf margin, abaxial. K, petiole. L, capsule. M, seed. D, G-L from FAA and dry sample, Saunders 3059; A-C, E-F from dry bulk population sample, Saunders \& Saddi 3063. Illustration: Y. Wilson-Ramsey.

base truncate, tomentose, pannose, stellate, stipitate-stellate, both faces glandular-capitate, short; adaxial stellate, glands pyriform. Leaves distichously arranged, evenly distributed, horizontal; petioles $3-18 \times 18-40 \mathrm{~mm}$, ridged; laminas concolorous, chartaceous, rumpled, surface not visible, usually elliptic, ovate, widely ovate, narrowly elliptic, oblong, oblong-ovate, 
rhombic, $5.6-15 \times 4.2-10 \mathrm{~cm}$, apex usually obtuse, acute to rounded, base subcordate $2-10 \mathrm{~mm}$, or rounded, obtuse, insertion planar, margins irregularly serrate, teeth $2-7 \times 2-4 \mathrm{~mm}$; adaxial yellow-olive, densely tomentose-velutinous, pannose-tomentose, stellate, stipitate-stellate, soft, slightly asperous; abaxial grayish brown, stipitate-ferrugineous tier longer, denser than on adaxial face, velutinous tier longer than on adaxial face; veins 5-7 lateral pairs above 5-7 basal palmate veins. Inflorescence axillary, crested, cymose, moderately dense clusters 1-3 cm, rarely lax in age, ca. 25-30-flowered. Bract stipe ca. $1 \mathrm{~mm}$; bracts 4 per floral pair, subequal, connate at $0.1-0.5 \mathrm{~mm}, 2.8-7 \times 0.8$ $1.8 \mathrm{~mm}$, narrowly lanceolate, tomentose, pannose, stellate, stipitate-stellate. Bracteoles 2-3, connate or distinct, very widely obtrullate, 3-5 mm. Pedicels absent. Flowers distylous, paired, ca. $3 \mathrm{~mm}$ diam. Calyx turbinate-clavate, 5-angled, 5-ribbed, 5-dentate, 4.5-7 × 2.5-4 mm; teeth ovate-triangular-mucronate, $0.8-2.5 \mathrm{~mm}$, interior villous, simple, rays $\geq 2$; tube interior sericeous, base nectary $0.5 \mathrm{~mm}$; exterior calyx tomentose, stellate, stipitate-stellate. Petals 5, golden yellow, spathulate, subconduplicate, recurved apex, obovate, 3.5-6.6(-7.1) × 1-2.5 mm, apical margin always deeply emarginate to $0.3 \mathrm{~mm}$, few-ciliate, trichomes $2-5$, simple, two rays, adaxial lamina midlength scant pilose, trichomes ca. $10,0.2-0.7 \mathrm{~mm}$, rays $1-2$, abaxial surface: few short trichomes, simple; claw 0.7$1.4 \mathrm{~mm}$, free from stamens. Thrum stamens 4.6$7.5 \mathrm{~mm}$, papillose; filaments 5 , subglabrous, long trichomes inside at base over glands; tube 1.5$2.4 \mathrm{~mm}$, rarely with two extra vestigial stamen filament bases; anthers $0.8-1.1 \mathrm{~mm}$. Thrum pistil dark, 3.6-4.8 mm; ovary 1.0-1.5 mm, densely hirsute, base less to subglabrous, stellate to two rays, base villulose, setose, glandular; style base inflexed, 1.3-3.1 mm, densely hirsute to stigma, stellate; stigma reflexed $20^{\circ}$, clavate-fimbriate, ovoid, yellow-green, 0.7-1.5 $\times 0.2-0.3 \mathrm{~mm}$, stipitate-stellate at base, stipes medium long, 0.3$0.6 \mathrm{~mm}$. Androgynophore $0.2 \mathrm{~mm}$, adnate with calyx base. Pin stamens $3-5 \mathrm{~mm}$; filaments 5 , adaxial red-mottled, appressed simple trichomes directed toward base in filaments and tube apex; tube $2.2-4 \mathrm{~mm}$, abaxial (rarely) with 1-3 setae between petal bases, simple, rays 2-3; anthers 0.8-1.1 mm, red papillose. Pin pistil 4.5-7.4 mm; ovary base subglabrous portion smaller than in thrums, trichomes at base, fewer, shorter; style inflexed, apex and stigma reflexed $45^{\circ}$; stigma presentation excentric, clavate-fimbriate, ovoid-ellipsoid, yellow-green, 0.7-1.3 $\times$ 0.2$0.9 \mathrm{~mm}$, base stellate, some stipitate-stellate, trichomes with long stipe. Capsule hidden in calyx, obovoid, 2.5-3.5 $\times 1.6-2.1 \mathrm{~mm}$, apex rounded, pubescent, setose, stellate to three rays, trichomes fewer, rays fewer to base; loculicidal dehiscence incomplete, mostly apical; chartaceous, apex slightly sclerified within. Seed brown monochromatic or white mottled-banded, obovoid, 1.8-2.8 × 1.3-1.8 mm, striate, foveate, minutely verrucose; raphe bicolored.

Common names. "Breixo" (Harley \& Souza $10015 \mathrm{~K})$, "Malva" (Ratter $863 \mathrm{UB})$.

Etymology. Waltheria matogrossensis is named for its provenance, Mato Grosso.

Distribution and habitat. Restricted to $\mathrm{CW}$ Brazil on the western edge of the Brazilian Plateau in eastern Mato Grosso, common from the Serra do Roncador, occurring centrally in Serra Azul near the upper Xingu watershed flowing to the Amazon River, and common on the western edges of the plateau in the Chapada Guimarães and São Vicente near Cuiabá at elevations of 450-600 (1500 at Serra Azul) m. a.s.l (Fig. 5). Towards the SW edge of the plateau bordering the Pantanal, it occurs near Rio Verde de Mato Grosso do Sul, and the Serra de Pimenteira. It is found in open grassy cerrado, rarely closed cerrado or campo rupestre, reformed or ecotones with dry forest, on the ridge of plateaus, hillsides, often associated with quartzite hills, sandstone hill formations or rock outcrops, in sandy soil, or sandy clay soil mixed with stone or over a rock basement.

Observations. Waltheria matogrossensis and $W$. coriacea are closely related. One plant, Hatschbach 66576, has lax inflorescences like $W$. coriacea, but appearing lax in age rather than soon lax. See comments under $W$. coriacea, but it can be separated from all species of 
$W$. ferruginea allies by its woolly tomentose leaves and the petal surface pilose, the apices always deeply emarginate, to $0.3 \mathrm{~mm}$, within a flower, and bracts with short glandular-capitate trichomes present on both sides.

Waltheria matogrossensis also shares on stem vestiture very long stipes in the ferruginea type stipitate-stellate trichome with $W$. biribiriensis, though stipes are to $1 \mathrm{~mm}$ in the latter species.

The name Waltheria polyantha K. Schum. has been misapplied to this species, even by Schumann on Riedel 976, and widely used, but examination of the type (Sello 1437 F!) shows that $W$. polyantha is a different species with a penicillate stigma endemic to the Serra Espinhaço in Minas Gerais (Serra Itambé and Diamantina). That name was also misapplied to an elongateplumose stigma species from Bolivia and Brazil on one example.

Flowering collections range year round, but are mostly from March through June, peaking in March and April. Flowers are diurnal, flowers are open until at least 5 pm (unpubl. obs., Saunders, 1995). Isoplethic population levels do occur, with six pins, six thrums (Saunders 3064). Floral visitors recorded during taxonomic fieldwork include wasps, bees, butterflies and two unidentified Curculionidae beetles (unpubl. obs., unpubl. data, Saunders, 1995). Floral tops are often chewed off in live flowers.

Waltheria matogrossensis is potentially conservation assessed as "of least concern" (LC).

\section{Specimens examined.}

Paratypes. BRAZIL. Mato Grosso. Munic. Água Boa: Serra do Roncador, ca. $86 \mathrm{~km} \mathrm{~N}$ of Xavantina, 4-VI-1966, Irwin et al. 16620 a (E, F, HB, MBM, MICH, MO, NY, RB, UB 2 sheets, W). Munic. Barra do Garças: ca. $35 \mathrm{~km}$ (straight line) ENE of Barra do Garças, 4-V1973, Anderson et al. 9708 (CTES, MO, R, UB); Serra do Azul, Morro das Antenas do Cristo, 20-VI-1988, Saddi 9125 (UFMT); Serra Azul, Clube da Maçonaria, ao longo camino para Cachoeira Corrego Lajedo, 28-V-1988, Saddi 9408 (UFMT); General Carneiro para Barra do Garças, BR-070, km 61-62, cerrado fechado de bambuzal, 16-III-1990 Saddi 11620 (UFMT); idem, 11622 (UFMT); idem 11623 (UFMT);
Serra Azul, circunvizinhança da cidade, ao longo estreada Antena do Cristo, desvio $1500 \mathrm{~m}$, SINDACTA, 17-III-1990, Saddi 11673 (UFMT); Serra Azul, circunvizinhança da cidade, ao longo Córrego Lajedo, desvio $2.3 \mathrm{~km}$, asfalto para SINDACTA, 17-III-1990, Saddi 11770 (UFMT); Barra do Garças para Novo Xavantina MT-158, $\mathrm{km} \mathrm{68}$, Valé do Sonho, Serra Azul, desvio a esquerda rodovia de $10 \mathrm{~km}, 23-\mathrm{IV}-1990$, Saddi 12259 (TEX, UFMT). Munic. Campinápolis: entre Nova Xavantina e Campinápolis, Placa Nativa, São Cristovã, 36-38 km na MT-251, Sope do Morro, 22-IV-1990, Saddi 12026 (UFMT). Munic. Chapado dos Guimarãres: S. de Chapada, V-1827, Riedel 976 (LE). Munic. Cuaibá: Salgadeira, Chapada dos Guimarães, 16III-1985, Filho 291 (UFMT); Rodovia CuiabáChapada dos Guiamarães, próximo a Salgadeira, 23-III-1982, Monteiro \& Leitão 19 (UFMT); Chapada dos Guimarães, Morro São Jerônimo, ente a Rodovia e Morro, ca. $4 \mathrm{~km}$ depois da Casa de Pedras, cerrado aberta, vale montanhoso, 25IV-1990, Saddi 12444 (CTES, TEX, UFMT); Chapada dos Guimarães, Morro São Jerônimo, ente a Rodovia e Morro, ca. $4 \mathrm{~km}$ depois da Casa de Pedras, todos especimes da mesma planta 25 IV-1990, Saddi 12446 (TEX, UFMT); CuiabáChapada dos Guimarães at km 60, Salgadeiro, $27-$ IV-1975, Saddi 1281 (UFMT); BR-364, camino para Casa de Pedras (Gruta de São Vicente, estrada São Lourenço, ca. $15 \mathrm{~km}$ do asfalto), 03IV-1983, Saddi 2596 (TEX, UFMT); BR-364 to Casa de Pedras (Route for São Vicente), turnoff to São Lorenço, $15 \mathrm{~km}$ from asphalt, disturbed cerrado along dirt track, 4-III-1990, Saunders \& Saddi 3056 (TEX, UFMT); Cuiabá-São Vicente, camino para Gruta, beira da estrada, $2 \mathrm{~km}$ da Sede para a Casa de Pedras, 04-III-1990, Saddi \& Saunders 11564 (TEX, UFMT); BR-364 to Casa de Pedras (Route for São Vicente), turnoff to São Lorenço, $15 \mathrm{~km}$ from asphalt, 4-III-1990, Saunders \& Saddi 3056 (TEX, UFMT); idem, Saunders \& Saddi 3057 (TEX, UFMT); idem, Saunders \& Saddi 3058 (TEX, UFMT); idem, Saunders \& Saddi 3059 (CTES, TEX, UFMT); Cuiabá-São Vicente, camino Casa de Pedras, depois de Sede da Fazenda, 1-2 km, 04-III-1990, Saunders \& Saddi 3060 (TEX, UFMT); idem, Saunders \& Saddi 3061 (TEX, UFMT); idem, 
Saunders \& Saddi 3062 (TEX, UFMT); Km 322323 on BR-364, 4-III-1990, Saunders \& Saddi 3063 (TEX, UFMT, US); 1 km before Salgadeira on road from Cuiabá, 5-III-1990, Saunders \& Saddi 3064 (TEX, UFMT); idem, Saunders \& Saddi 3065 (TEX, UFMT); $1 \mathrm{~km}$ past Salgadeira on road to Chapada dos Guiamarães, between Salgadeira and Porâo Inferno on road to Chapada dos Guimarães, 5-III-1990, Saunders \& Saddi 3066 (TEX, UFMT); idem, Saunders \& Saddi 3067 (TEX, UFMT); between loc at "1 km past Salgadeira on road to Chapada dos Guimarães between Salgadeira and Portão Inferno on road to Chapada dos Guimaraes" and loc. at "road to Morro do São Jeronimo between bridge of Casa de Pedra and the first fence of Estancia Planalto", 5-III-1990, Saunders \& Saddi 3068 (TEX, UFMT); road to Morro do São Jeronimo between bridge of Casa de Pedra and the first fence of Estancia Planalto, 5-III-1990, Saunders \& Saddi 3069 (TEX, UFMT); Cuiabá-São Vicente, camino para Gruta, beira da estrada, $2 \mathrm{~km}$ da Sede para a Casa de Pedras, 04-III-1990, Saddi \& Saunders 11564 (TEX, UFMT); idem, Saddi \& Saunders 11565 (UFMT); Cuiabá-São Vicente, camino Casa de Pedras, depois de Sede da Fazenda, 1-2 km, 04-III-1990, Saddi \& Saunders 1156711570 (TEX, UFMT); rodovia Emanoel Pinheiro, Salgadeira, $1 \mathrm{~km}$ antes do Terminal Turistico, imediações da placa do Rodovia, 05-III-1990, Saddi \& Saunders 11572-11573 (TEX, UFMT); rodovia Emanoel Pinheiro, Salgadeira, 05-III1990, Saddi \& Saunders 11574 (TEX, UFMT); entre Salgadeira e Porão do Inferno, $1 \mathrm{~km}$ em ambas margems, 05-III-1990, Saddi \& Saunders 11577 (TEX, UFMT); caminho para Morro São Jerônimo, entre Casa de Pedras e $1^{\circ}$ portéira Estância Planalto, 3 km adiante, 05-III-1990, Saddi \& Saunders 11578 (TEX; UFMT). Munic. General Carneiro: BR-70, $115 \mathrm{~km}$ W do Barra do Garças, 17-I-1989, Krapovickas \& Cristóbal 43008 (CTES, TEX); Paredão Grande, km 163165 on BR-070, desvio de $1 \mathrm{~km}$ para Fazenda São Sebastião, 16-III-1990 Saddi 11590 (UFMT); Idem, Saddi 11591 (UFMT); Paredão Grande, $\mathrm{km} \mathrm{163-165} \mathrm{on} \mathrm{BR-070,} \mathrm{desvio} \mathrm{de} 1 \mathrm{~km}$ para Fazenda São Sebastião, 21-IV-1990, Saddi 11905 (UFMT). Munic. Nova Maringá: entre Ponte de Pedra e Rio Sangue, IV-1918, Kuhlmann 2164 (R,
SP). Munic. Nova Xavantina: city of Xavantina on the Rio das Mortes, $1 \mathrm{~km} \mathrm{SSW}$ of center of city at base of low quartzite hill, $14^{\circ} 42 \mathrm{~S}, 52^{\circ}$ 21 'W, 27-VIII- 1968, Eiten \& Eiten 8430 (K, UB); Serra do Roncador, $7 \mathrm{~km} \mathrm{SW}$ of Xavantina, $\left(14^{\circ} 44 \mathrm{~S}, 52^{\circ} 20^{\prime} \mathrm{W}\right), 3-\mathrm{IV}-1968$, Ratter et al. 814 (E, K, MO, NY, RB, U). Munic. Pedra Petra: Serra da Petrovina, Rodoviaria BR-364, 16-V1995, Hatschbach et al. 62848 A (CTES). Munic. Ribeirão Cascalheira: $260 \mathrm{~km}$ along new road NNE of village of Xavantina ( $1 \mathrm{~km}$ due W of Royal Geographic Society Base Camp at $12^{\circ} 51$ 'S, $51^{\circ}$ $45 \mathrm{~W}$ ), along main road just $\mathrm{N}$ of turnoff road to Base Camp, 3-XII-1969, Eiten \& Eiten 9677 (SP, US); Royal Society/ Royal Geographical Society Expedition 1967-1969 Base Camp: $\left(12^{\circ} 49\right.$ 'S, $51^{\circ}$ 46 W), 1967-1969, Gifford 36 (CTES); $10 \mathrm{~km} \mathrm{~S}$ of base camp $\left(12^{\circ} 54 \mathrm{~S}, 51^{\circ} 22 \mathrm{~W}\right), 270 \mathrm{~km} \mathrm{~N}$ of Xavantina, 10-III-1968, Gifford 80 (K); $6 \mathrm{~km}$ N of Royal Society/Royal Geographical Society Expedition 1967-1969 Base Camp: $12^{\circ} 49$ 'S, 51 ${ }^{\circ}$ 46 'W, 14-IX-1968, Harley \& Souza 10015 (K, MO, NY, RB, UB); Serra do Roncador, ca. 210 Km N de Xavantina, near Rio Turvo, 28-V-1966, Irwin et al. 16172 (CTES, MBM, MO, NY, UB, UPS); Xavantina-Cochimbo Expedition, near the base camp of the Anglo/Brazilian Northern Mato Grosso Expedition 1967/69 Base Camp (12 54 S, $\left.51^{\circ} 52 \mathrm{~W}\right)$, ca. $270 \mathrm{~km} \mathrm{~N}$ of Xavantina, 10XI-1967, Ramos \& Sousa 137 (CTES); Serra do Roncador, near Anglo/Brazilian Northern Mato Grosso Expedition 1967/1969 Base Camp (12 $54 \mathrm{~S}, 51^{\circ} 52 \mathrm{~W}$ ), (close to the Xavantina-São Felix road, ca. $275 \mathrm{~km} \mathrm{~N}$ of Xavantina), 6-IV1968, Ratter et al. 863 (E, K, MO, NY, RB, UB); Serra do Roncador, $8 \mathrm{~km}$ NE of Anglo/ Brazilian Northern Brazilian Mato Grosso Expedition 1967/1969 Base Camp (12 54 'S, $51^{\circ} 52$ 'W), 9-IV-1968, Ratter et al. 914 (NY, RB, UB). Munic. Sto. Antonio do Leverger: 8 km de BR-364, camino a Aguas Quentes, 30-I1989, Krapovickas \& Cristóbal 43147 (CTES, TEX). Mato Grosso do Sul. Munic. Rio Verde: Rodoviaria Campo Grande-Cuiabá, 15-V-1973, Hatschbach 31924 (C, CTES, F, INPA, MBM, MO, NY, US); Serra da Pimenteira, 8-II-1975, Hatschbach et al. 35955 (CTES, MBM); Rio Verde, Sete Quedas, 8-VIII-1997, Hatschbach et al. 66576 (CTES). 


\section{Key to sections of Waltheria (modified from Schumann, 1886)}

1. Capsule dehiscence operculate; stipule base ovate or subcordate on one or both sides; stem trichomes simple, stellate absent or very rarely at plant base sect. Stegowaltheria K. Schum.

1. Capsule dehiscence loculicidal; stipule base truncate; stem trichomes stellate (rarely only stellate at plant apex, some W. communis) sect. Waltheria

\section{Key to the species of Waltheria ferruginea allies}

1. Inflorescence axillary, subterminal in distal 7-10 nodes, often hidden in erect or ascending spirally arranged leaves; short glandular-capitate trichomes on adaxial calyx .............................................................................W. terminans 1. Inflorescence axillary, crested, usually erect over distichously arranged horizontal, reclining, or pendulous leaves; glandular-capitate trichomes absent on adaxial calyx 2 2(1). Most leaves non-lanceolate, rather ovate, elliptic, oblong, oblong-obovate, or very variable along branch or between plants 3

2. Most leaves lanceolate, oblong-lanceolate, narrowly ovate, narrowly elliptic or narrowly oblong ........................ 4 3(2). Stem apices densely ferrugineous-tomentose; leaves coriaceous, discolorous, ca. 2-8 $\times 2-4 \mathrm{~cm}$, variable, usually ovate-oblong or elliptic, adaxial surface dark green, ferrugineous tomentellous soon pubescent, glabrescent; inflorescences soon very lax; petal apices rounded or shallowly emarginate

W. coriacea 3. Stem apices woolly-tomentose; leaves chartaceous, concolorous, ca. 6-15 $\times 4-10 \mathrm{~cm}$, elliptic, ovate, widely ovate, adaxial surface densely velutinous; inflorescence in clusters, rarely lax in age; petal apices always deeply emarginate

W. matogrossensis 4(2). Leaf laminas on distal stem discolorous, adaxial surface soon visible; stem internodes 6-55 mm ................... 5

4. Leaf laminas on distal stem concolorous, surfaces not visible; stem internodes 2-20 mm ................................... 8 5(4). Leaves very discolorous, adaxial edges pale-rimmed and villous, heterotrichous from trichomes different at margins than those on face inside margin, young lamina bases cuneate, base slightly canaliculate, insertion acuteangled W. ferruginea s. str. 5. Leaves on distal stem discolorous, homotrichous on face and margins, at times pale rimmed ( W. biribiriensis); lamina bases rounded, subcordate, rarely cuneate, insertion obtuse-angled or planar

6(5). Stem apices and leaves woolly-tomentose from dense stipitate-stellate trichomes, stipes long (to $1 \mathrm{~mm}$ ); bracts distally fimbriate-laciniate (antler-like), rarely 1 bract entire W. biribiriensis 6. Stem apices and leaves tomentose or tomentellous, stipitate-stellate trichomes short, stipes 0.1 to $0.4 \mathrm{~mm}$; bracts lobed, dentate, or entire .....

7(6). Leaf lamina adaxial surface evenly glabrescent, abaxial surface incanus (whitish) tomentose; petals (1.6-)1.8-2.7 mm wide; calyx 3.5-4.2 mm wide; calyx with long (to $1 \mathrm{~mm}$ ), thin rayed stellate-velutinous overlayer, long at base progressively shorter to apex; seed calyptrate, not 5-lobulate in apical view; pin style geniculate at base; shrubs to $4 \mathrm{~m}$ tall; caatinga W. brachypetala 7. Leaf lamina adaxial leaf surface floccose-glabrescent, abaxial surface yellow-green tomentellous; petals 1.2-1.7 mm wide; calyx (1.8)2.5-3.5 mm wide; calyx tomentose, tomentellous; seed not calyptrate, 5-lobulate in apical view; pin style not geniculate at base; shrubs to $2 \mathrm{~m}$; cerrado W. flavovirens 8(4). Leaf laminas slightly asperous, adaxial surface ferrugineous-tinged olive, bases rounded, subcordate for 2-4 mm, 9 palmate basal veins; bracts $6.2-10 \mathrm{~mm}$; thrum stamens $5.3-5.8 \mathrm{~mm}$ W. hatschbachii 8. Leaf laminas very soft, both surfaces pale gray-olive, bases rounded, 5 palmate basal veins; bracts 2.4-3.5 mm; pin pistil $8.9 \mathrm{~mm}$

W. hoehnei

\section{DISCUSSION AND CONCLUSIONS}

All nine species of the Waltheria ferruginea allies presented in the key share the ferruginea type stipitate-stellate trichome, illustrated here (Fig. 1B, C), and earlier drawn for $W$. ferruginea by the author in Cristóbal \& Saunders (2006: 112, Fig. 2F4). The ferruginea type stipitate-stellate trichome differs from other stipitate-stellate trichomes in the genus by its stipe being deciduous, weak, often breaking above base rather than stipes thick, persistent, their stellate rays in some types deciduous. 
These new species are placed in the section Waltheria, and belong to the clavate-stigma species group, one of the three pin stigma type informal groups described for Waltheria species (Saunders, 1993). Clavate-stigma species have a clavate-fimbriate stigma present in pin flowers, rarely clavate-papillose in thrum flowers. Other groups are the elongate-plumose stigma species group, and penicillate-stigma species group (Saunders, 1993; 1995; 2005; 2007; Cristóbal \& Saunders 2006; Saunders \& Pozner, 2007), more broadly named but all mostly congruent with Schumann's stigma type groups (Schumann, 1886), or Waltheria pyrolifolia A. Gray, with a simple 2-lobulate stigma (Saunders, 2011). For seven Brazilian species of Grão Mogol (Cristobal \& Saunders, 2006), these types are illustrated clearly for application of types fide Saunders 1993 but text types are different than names used in 1993 or now by the author: substitute penicillate s. 1. (fan-plumose) for capitate ( $W$. aspera $\mathrm{K}$. Schum., $W$. indica L.), and clavate-fimbriate for penicillate ( $W$. ferruginea).

\section{New Taxonomic Characters for Waltheria}

New taxonomic grouping characters here reported for Waltheria are: subconduplicate petals found in some species of the genus (Fig. 1A) vs. flat petals used in keys to all the tribe; ferruginea type stipitate-stellate trichomes (Figs. 1B, C); stipitatestellate trichomes with short to medium long stipes on the style just below the stigma or present in the stigma base (Figs. 4A, B); pin stigma exserted presentation excentric (Fig. 6M); capsules hidden in the calyx previously more clearly illustrated in Saunders (1993:367, Fig. 9 1, m); papillose stamens; and differently shaped sessile glandular trichomes on stipules between species.

Other characters found in this work appear to be unique to a particular species: rugulose seeds (Waltheria hoehnei); minute glandular trichomes on adaxial petals ( $W$. hoehnei); reduced androgynophores woody (W. hatschbachii); different ovary vestiture between pin and thrum flowers ( $W$. matogrossensis); downward pointing trichomes on adaxial stamen tube base over glandular areas (W. matogrossensis); glandular areas on abaxial petal bases, and on adaxial stamen tubes, possibly nectiferous ( $W$. matogrossensis).
Presence of trichomes on calyx tube interiors described here for all the species was first reported by Cristóbal (1983) for W. carpinifolia A. St.-Hil. \& Naudin.

These and other new characters recently found that were not mentioned here will be presented in another more profound work with a new phylogenetic analysis for these allies to better estimate their relationships and evolution.

Biogeographical Intercontinental Disjunctions

Regarding Waltheria flavovirens, found in but not limited to the Chapada dos Veadeiros, Waltheria appears to be another example of a genus with an enigmatic biogeographical connection between the Chapada dos Veadeiros, Africa and Australia. This pattern was earlier described for Triraphis R. Br. (Poaceae) from campo rupestre by Filgueiras (2002: 124), citing Filgueiras \& Zuloaga (1999). Waltheria has paleotropical endemics in Australia, Africa, Madagascar, tropical Asia and Polynesia (Saunders, 2011). Waltheria virgata Ewart \& Cookson is from NW Australia, in spinifex steppe savanna, peripatric to its closest generic relative, Dicarpidium F. Muell. from the Kimberley region (Saunders, 1995; Florabase Australia). Waltheria lanceolata $\mathrm{R}$. Br. ex Mast. from West Africa, is in the clavate stigma species group (Saunders, 1993) with the species of $W$. ferruginea allies in Brazil, including $W$. flavovirens from Chapada dos Veadeiros, and surrounding areas (Fig. 5). Thus, the genus Waltheria shows a similar biogeographic connection between there, Africa and Australia as reported for Triraphis.

The above biogeographical connection makes even more interesting the observation of rugulose seeds in Waltheria hoehnei, only previously reported in the tribe for the Australian Dicarpidium. This is being further studied but may invalidate the previous use of subtuberculate, verrucose seeds to distinguish sect. Stegowaltheria from sect. Waltheria, with smooth seeds (Saunders, 1993; 1995; 2007). Schumann (1886) in the key to sections, described seeds as "glabra" for sect. Waltheria (as Euwaltheria) to separate them from sect. Stegowaltheria, with subtuberculate, verrucose seeds (see Saunders, 1995: 222; Saunders 2007: 159-160). 
This is not stated in Schumann's key as a contrasting character, but can be inferred from the species description for $W$. macropoda Turcz. seeds (sect. Stegowaltheria), that he described as subtuberculate.

The stipitate-stellate trichomes with short to medium long stipes on the style apex or stigma base of $W$. coriacea and $W$. matogrossensis are unique in the Hermannieae (Figs. 4A, B, respectively). However, the Australian tribe Lasiopetaleae, close to Hermannieae (Whitlock et al., 2001), has two species of Lasiopetalum Sm. with large, prominent bizarre downward pointing stipitate-stellate trichomes distributed from the style base nearly to the apex or just near the apex (Jenny, 1985: Figs. 1, 4, 30, 31, 34; Jenny, 1988: 231; Whitlock 2001: 424). It is not known if these are homologous between genera.

\section{Waltheria Diversity Including the New Species}

The validation of these five new species from Brazil completes the publication of all of the seven new Brazilian Waltheria designated in a dissertation monograph (Saunders, 1995). In that work, 60 species were recognized, half of them occurring in Brazil, those all treated but for $W$. indica L. It became the basis for the Flora of Brazil checklist, cited therein earlier as a general reference prior to the published version (Esteves, 2010), cited also in the official flora website (see below). In the dissertation monograph, twentytwo species were considered to be endemic to Brazil, twice more than the nine species endemic to Mexico mostly from the Transvolcanic Belt of the Sierra Madre (Saunders, 1993). Within Brazil, Minas Gerais had the highest diversity and endemism, with 19 species in all, six of them endemic to the Serra Espinhaço Range, including $W$. hatschbachii, $W$. biribiriensis, $W$. terminans and $W$. ferruginea s. str. from the $W$. ferruginea allies (Fig. 5). Bahia had 16 species, Mato Grosso and Tocantins both had seven species of Waltheria, and Goiás had four species. Currently, the website for the flora of Brazil (accessed $3 \mathrm{Dec}$ 2020) lists a total of 27 Waltheria species (http:// floradobrasil.jbrg.gob.br/reflora/listaBrasil), not including these five new ones as published, but including four other newly described species, and one range extension to Brazil.

\section{ACKNOWLEDGMENTS}

The author is grateful to the directors of the Instituto de Botánica Darwinion (IBODA-CONICETANCEFN), and the Billie L. Turner Plant Resources Center in Austin for facility support, Yevonn WilsonRamsey and Francisco Rojas for illustrations, Jack Neff and Phil de Vries for insect identifications, Steve Ginsbarg, Eugenia Gullace for translations, Ramon Palacios for the spanish abstract, Nilber Gonçalves de Silva for his generosity in locating new material, providing photographs and permission for their use for W. hoehnei, the NSF for grant BSR-8800899, CNPq of Brazil for sponsorship of fieldwork in Brazil, George Eiten $\dagger$, Andre M. de Carvalho $\dagger$, Anna M. Giulietti, Ariane \& Oswaldo Peixoto, Gert Hatschbach $\dagger$, Jefferson Prado, Cintia Kameyama, and Nagib Saddi for their valuable time given for it, and helpful instruction on vegetation, Egon Köhler for providing pollen voucher references for a future collaboration on pollen, George Yatskievych for correcting $W$. hoehnei and Amber Horning for tracking down isotype exchange localities, Regina Célia de Oliveira, Susan Lutz, Olof Ryding, Maria Mamede for type information. To the anonymous reviewers and to Manuel Belgrano, Christian Zanotti and Fernando Biganzoli for very helpful comments, Roberto Donadio for composing the plates, and all for greatly improving the manuscript. The author is extremely indebted and very grateful to Carmen Cristobal†, Beryl Simpson, Ramón Palacios, and Fernando Zuloaga for their support. Finally, the author thanks the directors of the herbaria cited for loans or personal access to samples.

\section{BIBLIOGRAPHY}

Amorim, B. S.; J. G. Saunders, A. L. Du Bocage Neta \& M. Alves. 2009. Malvaceae s. l., in M. Alves, M. de Fatima Araujo, J. R. Maciel \& S. Martins (eds.), Flora de Mirandiba, pp. 243-260. Recife: Associação Plantas do Nordeste.

Coutinho, T. S.; M. Colli-Silva \& M. Alves. 2020. Novelties in Brazilian Waltheria L. (Byttnerioideae, Malvaceae): two new species and one re-establishment. Acta Botanica Brasilica 34(3): epub. DOI: https://doi.org/10.1590/010233062020abb0088

Cristóbal, C. L. 1983. Esterculiaceas, in R. Reitz (ed.), Flora ilustrada Catarinense. Parte I. As Plantas: fasc. ESTE:1-57. Est. 1-10, IOSCE, Itajai, Santa Catarina. 
Cristóbal, C. L. \& J. G. Saunders. 2006. Sterculiaceae, in J. R. Pirani, A. M. Giulietti, R. de Mello-Silva, A. Rapini, L. Cordero, L. P. de Queiroz, and D. C. Zappi (eds.), Flora de Grão Mogol, Minas Gerais. Parte 3. Angiospermas P-T. Vol. 24, pp. 107-113. Boletim de Botanica. São Paulo: Universidade de São Paulo. DOI: https://doi.org/10.11606/ issn.2316-9052.v24i1p107-113

Esteves, G. 2010. Waltheria, in R.C. Forzza et al. (eds.), Catalogo de plantas e fungos de Brazil. Vol. 2, pp. 12251226. Rio de Janeiro, Andrea Jakobsson Estudio.

Filgueiras, T. S. 2002. Herbaceous plant communities, in P. S. Oliveira \& R. J. Marquis (eds.), The cerrados of Brazil: ecology and natural history of a neotropical savanna, pp. 121-139. New York: Columbia University Press. DOI: https://doi.org/10.7312/oliv12042-008

Filgueiras, T. S. \& F.O. Zuloaga 1999. A new Triraphis (Poaceae: Eragrostideae) from Brazil: The first record of a native species in the New World. Novon 9: 36-41. DOI: https://doi.org/10.2307/3392114

Florabase Australia website: http://auh/ala.org.au/species/ urn/:1sid:biodiversity.org.au:apr.taxon

Hensold, N. 1988. Morphology and systematics of Paepalanthus subgenus Xeractis (Eriocaulaceae). Systematic Botany Monographs Vol. 23: 1-50.

Hoehne, F. C. 1939. Plantas e Substâncias Tóxicas e Medicinais. Rio de Janeiro: Graficars. Pp.191-193.

IUCN Red List Categories: Version Aug. 14, 2019. IUCN Species Survival Commission, IUCN, Gland, Switzerland and Cambridge, UK.

Jenny, M. 1985. Funktion und systematische bedeutung des gynoeciums bei Sterculiaceen. Ph. D. thesis, Universität Zurich.

Jenny, M. 1988. Different gynoecium types in Sterculiaceae: Ontogeny and functional aspects, in P. Leins, S. C. Tucker \& P. K. Endress (eds.): Aspects of Floral Development, pp. 225-236. Berlin: Cramer.

Köhler, E. 1976. Pollen dimorphism and heterostyly in the genus Waltheria L. (Sterculiaceae), in I.K. Ferguson \& I. Mueller (eds.). The evolutionary significance of the exine pp. 147-161. London, Linn. Soc. Symp. Series No. 1.

Lawrence, G. H. M. 1951. Taxonomy of Vascular Plants. New York: Macmillan. $823 \mathrm{pp}$.

Medeiros, M. B. de; B. M. Teles Walter, G. P. da Silva, B. M. Gomes, I. L. P. Lima, S. R. Silva, P. Moser, W. L. Oliveira \& T. B. Cavalcanti. 2012. Vascular flora of the Tocantins river middle basin. Check List 8(5): 852-885. DOI: https:// doi.org/10.15560/8.5.852

Moulton, J.C. 1908. The collections of William John Burchell D. C. L. in the Hope Department Oxford University Museum. IV. On the Lepidopteran Rhopalocera collected by
W. J. Burchell in Brasil 1825-1830. Annals and Magazine of Natural History, sér 8, 2(8): 165-195. DOI: https://doi. org/10.1080/00222930808692467

Oliveira-Filho, A. T. \& J. Ratter. 2002. Vegetation physiognomies and woody flora of the cerrado biome, in P. S. Oliveira \& R. J. Marquis (eds.), The cerrados of Brazil: ecology and natural history of a neotropical savanna, pp. 91120. New York: Columbia University Press. DOI: https:// doi.org/10.7312/oliv12042-007

Pohl, R. W. 1965. Dissecting equipment and materials for the study of minute plant structures. Rhodora 67: 95-96.

Radford, A. E.; W. C. Dickison, J. R. Massey \& C. R. Bell. 1974. Vascular Plant Systematics. Harper \& Row, Publishers, New York. $891 \mathrm{pp}$.

Saunders, J. G. 1993. Four new distylous species of Waltheria (Sterculiaceae) and a key to the Mexican and Central American species and species groups. Systematic Botany 18: 356-376. DOI: https://doi.org/10.2307/2419409

Saunders, J. G. 1995. Systematics and Evolution of Waltheria (Sterculiaceae: Hermannieae). Ph. D. Diss. The University of Texas, Austin. Available at PROQUEST, Ann Arbor, Michigan.

Saunders, J. G. 2005. New species of Waltheria (Hermannieae, Byttnerioideae, Malvaceae) from Paraguay, Argentina and Venezuela, and two new records of Waltheria for Paraguay.. Darwiniana 43: 201-211.

Saunders, J. G. 2007. Sterculiaceae of Paraguay II. Waltheria. Bonplandia (Corrientes) 16: 143-180. DOI: https://doi. org/10.30972/bon.161-2182

Saunders, J. G. 2011. Resurrection of the Maui endemic Waltheria pyrolifolia (Sterculiaceae, Hermannieae). Darwiniana 49(1): 76-85.

Saunders, J. G. \& R. E. Pozner. 2007. A new penicillatestigma species of Waltheria (Sterculiaceae, Hermannieae) endemic to Belize. Novon 17: 79-86. DOI: https://doi. org/10.3417/1055-3177(2007)17[79:ANPSOW]2.0.CO;2

Schumann, K. 1886. Sterculiaceae, in C.F. P. Martius, A. G. Eichler \& I. Urban (eds), Flora Brasiliensis, Vol. 12(3), pp. 1-114, plates 1-24. Monachii, Pilsiae: Apud. Fried. Fleischer in Comm. Facsimile reprint 1967 by Verlag von Cramer, Lehre, Germany.

Thiers, B. [continuously updated, accessed 2020] Index Herbariourum: a global directory of public herbaria and associated staff. New York Botanical Garden's Virtual Herbarium. Available from http://sweetgum.nybg.nybg.org/ih

Whitlock, B.; C. Bayer \& D. A. Baum. 2001. Phylogenetic relationships and floral evolution of the Byttnerioideae ("Sterculiaceae" or Malvaceae s. 1.) based on sequences of the chloroplast gene ndhF. Systematic Botany 26(2): 420-437. 